\title{
BIOLOGÍA FLORAL, VISITANTES FLORALES Y SISTEMA REPRODUCTIVO DE NYMPHAEA GRACILIS ZUCC. (NYMPHAEACEAE)
}

\section{FLORAL BIOLOGY, FLORAL VISITORS, AND REPRODUCTIVE SYSTEM OF NYMPHAEA GRACILIS ZUCC. (NYMPHAEACEAE)}

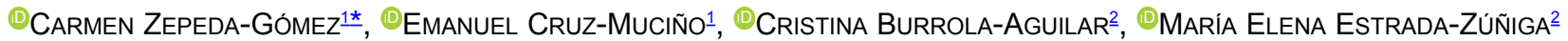

1Universidad Autónoma del Estado de México, Facultad de Ciencias, Toluca, Estado de México, México.

${ }^{2}$ Universidad Autónoma del Estado de México, Centro de Investigación en Recursos Bióticos, Toluca, Estado de México, México.

*Autor para correspondencia: zepedac@uaemex.mx

\section{Resumen}

Antecedentes: La biología floral influye en el sistema reproductivo de las plantas y permite identificar factores que afectan la supervivencia y dinámica de poblaciones vegetales.

Preguntas: ¿Cuáles son los atributos florales, el despliegue floral, el sistema y rendimiento reproductivo, y el principal visitante floral de $N$. gracilis? ¿Los cruces intrapoblacionales pueden conducir a una depresión por endogamia?

Especie de estudio: Nymphaea gracilis una hidrófita enraizada emergente endémica del centro de México y amenazada de extinción.

Sitio y años de estudios: El estudio se realizó en dos cuerpos de agua del municipio de Toluca, Estado de México. De agosto a diciembre de 2017 y 2018.

Método: La morfología floral y la función masculina y femenina se evaluaron a lo largo de la antesis. Se analizó la producción y biomasa de frutos y semillas, los visitantes florales y la relación polen:óvulo. La estrategia reproductiva se evaluó por experimentos de exclusión de polinizadores y polinizaciones artificiales.

Resultados: Nymphaea gracilis presenta antesis diurna de 4-6 días, receptividad estigmática los dos primeros días, protoginia incompleta y xenogamia facultativa. Apis mellifera realizó la mayor contribución de polinización. Los cruzamientos artificiales indicaron que la especie no es agamosperma y que mantiene un sistema de cruzamiento mixto, autocompatibilidad, autogamia, limitación de polen mediada por polinizadores y niveles relativamente bajos de depresión por endogamia.

Conclusión: El sistema mixto de reproducción en N. gracilis podría estar favoreciendo la permanencia de la población y garantizar la descendencia en un ambiente donde la disponibilidad de polinizadores es baja.

Palabras clave: autocompatibilidad, autogamia, depresión por endogamia, estrategia reproductiva, xenogamia facultativa.

\begin{abstract}
Background: Floral biology influences the plant reproductive systems and allows identify factors that affect the survival and dynamics of plant populations.

Questions: What are the floral attributes, the floral display, the reproductive system, reproductive performance, and the main floral visitors of $N$. gracilis? Can intrapopulation crossings lead to inbreeding depression?

Study species: Nymphaea gracilis an emerging rooted hydrophyte endemic to central Mexico and threatened with extinction.

Site and years of studies: The study was carried out in two bodies of water in the municipality of Toluca, State of Mexico. From August to December 2017 and 2018.

Methods: The floral morphology and the male and female function were evaluated throughout the anthesis. The production and biomass of fruits and seeds, the floral visitors and the pollen: ovule relationship were analyzed. The reproductive strategy was evaluated by pollinator exclusion experiments and artificial pollinations.

Results: Nymphaea gracilis presents diurnal anthesis for 4-6 days, stigmatic receptivity first two days, incomplete protogyny and facultative xenogamy. Apis mellifera made the largest contribution of pollination. Artificial crosses indicated that the species is not agamosperm and that it maintains a mixed crossing system, autocompatibility, autogamy, pollinator-mediated pollen limitation, and relatively low levels of inbreeding depression.

Conclusions: The mixed reproductive system in $N$. gracilis could be favoring the permanence of the population and guarantee the offspring in an environment where the availability of pollinators is low.
\end{abstract}

Key words: autogamy, facultative xenogamy, inbreeding depression, reproductive strategy, self-compatibility. 
La biología floral influye en el sistema reproductivo de las plantas, en el tipo de polinizador y en la transferencia de polen por los polinizadores (Lloyd \& Schoen 1992, Harder \& Barrett 2006). Por lo tanto, el estudio de atributos como tamaño y forma de la flor, despliegue y longevidad floral, tiempo de receptividad estigmática y maduración de las anteras, permiten comprender mejor la biología reproductiva de una planta (Primack 1985) e identificar algunos factores que afectan la supervivencia, dispersión, mantenimiento y regeneración de sus poblaciones (Schemske et al. 1994, Rodríguez-Pérez 2005). Esto es particularmente relevante en especies de distribución restringida y/o con amenazas de extinción debido a que permite visualizar alternativas de manejo, conservación o reintroducción (Schemske et al. 1994, Navarro \& Guitián 2002, Rodríguez-Pérez 2005, Castro et al. 2008).

El género de plantas acuáticas Nymphaea es el más diverso de la familia Nymphaeaceae, agrupa más de 50 especies con numerosos híbridos y variedades distribuidos en regiones tropicales y templadas de todo el mundo (Wiersema 1988, Borsch et al. 2011). Está dividido en cinco subgéneros de plantas con flores bisexuales, principalmente protóginas, y con antesis diurna o nocturna que dura de 2 o hasta 7 días (Wiersema 1988, Borsch et al. 2011). En las flores protóginas hay una separación temporal entre la fase femenina y masculina asociada a movimiento florales de apertura y cierre; típicamente el primer día la flor abre siendo funcionalmente femenina y en días posteriores volverá abrir, pero funcionalmente masculina (Capperino \&

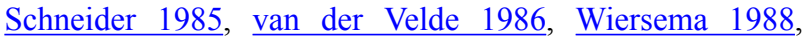
Orban \& Bouharmont 1995, Barrios \& Ramírez 2008, Endress 2010, Williams et al. 2010, Maia et al. 2014, Povilus et al. 2014). Las flores protóginas tienen un mayor éxito reproductivo femenino al reducir su interacción con el sexo masculino y aumentar las tasas de fecundación cruzada (Çetİnbaş \& Ünal 2014). Sin embargo, en el género Nymphaea existen excepciones a este patrón general, algunas especies son homógamas (Endress 2001) y al menos 10 utilizan la autogamia como alternativa reproductiva para sobrevivir en ambientes desfavorables; la mayoría de las especies autógamas son de distribución tropical y cuatro corresponden al subgénero Brachyceras (Conard 1905, Wiersema 1988, Povilus et al. 2014).

Nymphaea gracilis pertenece al subgénero pantropical Brachyceras y de las doce especies de ninfas presentes en México es la única endémica al territorio nacional (Lot et al. 1999, Zepeda-Gómez 2017). Según las leyes mexicanas se encuentra amenazada de extinción (SEMARNAT 2010) debido a la destrucción y fragmentación de su hábitat ( $\underline{\text { Lot }}$ \& Zepeda 2009). Naturalmente se distribuye en los estados de Aguascalientes, Ciudad de México, Durango, Guanajuato, Jalisco, México, Michoacán y Oaxaca (Lot et al. 1999, Novelo \& Bonilla-Barbosa 1999, Zepeda-Gómez
2017), pero la mayor parte de su distribución se ubica en la Cuenca del Río Lerma, una de las cuencas más importantes de México por su gran extensión, riqueza natural, histórica $\mathrm{y}$ social, pero sobre todo por sus graves problemas ambientales (GEM 2011).

Las poblaciones de $N$. gracilis son aisladas y pequeñas, es probable que esto se asocie a las limitaciones espaciales que impone el hábitat acuático, a la precaria interacción entre cuerpos de agua y a las actividades antrópicas prevalecientes en la Cuenca de Lerma, aunque es complicado determinar la magnitud y el efecto de cada una. Hasta ahora, no se han realizado estudios que aborden la biología o evaluaciones detalladas del estado de conservación de las poblaciones silvestres de $N$. gracilis, no obstante, el análisis de su biología floral permitirá comprender mejor su capacidad reproductiva, sus interacciones de polinización y contribuir a desarrollar estrategias que incrementen las probabilidades de su supervivencia futura.

El objetivo de este estudio fue investigar el despliegue floral, los principales visitantes florales y la estrategia reproductiva de $N$. gracilis en condiciones naturales, examinando los rasgos reproductivos más relevantes y discutiendo las posibles implicaciones en la conservación de esta hidrófita. En particular se pretende responder a las siguientes preguntas: (1) ¿Cómo es la morfometría y el despliegue floral de $N$. gracilis durante la antesis? (2) ¿Cuáles son los visitantes florales y el principal vector de polinización de la especie? (3) ¿Cuál es el sistema reproductivo y cómo es la producción de frutos y semillas de $N$. gracilis bajo diferentes tratamientos de polinización? y (4) ¿Es posible que los cruces intrapoblacionales conduzcan a una depresión por endogamia? Se espera que $N$. gracilis muestre estrategias dirigidas a favorecer la autofecundación, como se ha observado en otras especies neotropicales del subgénero Brachyceras (Prance \& Anderson 1976, Povilus et al. 2014).

\section{Materiales y métodos}

Especie de estudio. Nymphaea gracilis es una hidrófita enraizada emergente conocida popularmente como estrella de agua o zazamol, crece en lagunas, lagos, presas, canales, charcas temporales y áreas inundables poco profundas de zonas templadas de México, desde los 1,370 hasta los 2,600 m snm. (Lot et al. 1999, Novelo \& Bonilla-Barbosa 1999, Zepeda-Gómez 2017). Presenta hojas con láminas orbiculares de aproximadamente $40 \mathrm{~cm}$ de diámetro, flotantes sobre la superficie del agua y sostenidas por peciolos de hasta $50 \mathrm{~cm}$ de longitud. La mayoría de las plantas obtienen la madurez sexual después de 1 o 2 años y luego florecen cada año. Debido a la belleza estética de sus grandes y vistosas flores es una planta deseable para el 
cultivo en jardines acuáticos. Sus rizomas conocidos como atzatzamolli, zazamol o cabeza de negro, son comestibles y en ciertas regiones además se utilizan como forraje o para elaborar artesanías (Lot et al. 1999, Novelo \& BonillaBarbosa 1999, Zepeda-Gómez 2017).

Zona de estudio. El estudio se realizó durante agostodiciembre de 2017 y 2018 en dos cuerpos de agua de la localidad de San Cayetano Morelos, Municipio de Toluca, México (N 19² 24' 25", O 99 41' 11" y N 19² 24' 13", O $99^{\circ}$ 41' 17"). Ambos cuerpos de agua cubren una extensión aproximada de 9.5 ha (7 y 2.5 ha respectivamente), la distancia entre ellos es de $150 \mathrm{~m}$ y se ubican a 2,607 $\mathrm{m} \mathrm{snm}$. La región presenta un clima templado subhúmedo con lluvias de mayo a octubre y promedio de precipitación anual de 800-1,000 $\mathrm{mm}$. En la época lluviosa la profundidad máxima de los cuerpos de agua es de $3 \mathrm{~m}$ y cerca del $50 \%$ de su área se cubre de plantas acuáticas como Nymphoides fallax Ornduff, Polygonum spp., Nymphaea gracilis, Lemna spp. y diversos pastos y ciperáceas. Durante la época de estiaje el nivel de agua disminuye $30 \%$ o más.

Morfometría y producción de polen y óvulos. Para determinar los atributos morfológicos y dimensiones de las flores de $N$. gracilis, durante la época de floración (agostonoviembre) se colectaron en campo 30 flores de diferentes plantas completamente maduras y distribuidas a lo largo del cuerpo de agua. En cada flor se contó el número de pétalos, sépalos, estambres, carpelos y óvulos. Adicionalmente, y con la ayuda de un vernier digital, en cada flor completamente abierta se midió: 1) diámetro externo, 2) diámetro interno, 3) longitud del pedúnculo floral, 4) longitud de estambres y 5) longitud de apéndices carpelares (Figura 1). Además, se aplicó la técnica del rojo neutro al $0.1 \%$ (Vogel 1990) para determinar el sitio de emisión de olor.

Para determinar la cantidad de polen que produce una flor y la diferencia de polen producido entre anteras, todos los estambres de 30 flores se contaron y clasificaron en tres categorías en base a la secuencia de su dehiscencia (externos ( $>2.2 \mathrm{~cm}$ de longitud), intermedios $(\geq 1.3 \mathrm{~cm} \mathrm{y}$ $\leq 2.2 \mathrm{~cm}$ de longitud) e internos ( $<1.3 \mathrm{~cm}$ de longitud). El polen de un estambre de cada categoría y de 10 flores diferentes se colocó por separado en $0.5 \mathrm{ml}$ de azul de anilina en lactofenol y bajo el microscopio óptico se contó el número de granos de polen en tres alícuotas. El número de granos de polen por antera se determinó como el promedio de tres alícuotas multiplicadas por el factor de dilución (Kearns \& Inouye 1993). El número de granos de polen por tipo de antera (interna, intermedia y externa) se comparó mediante una ANOVA de un factor en el programa SPSS (IBM 2015). El número de granos de polen por flor se estimó multiplicando el número de granos de polen por antera por el número promedio de anteras por categoría y por flor.

El número de óvulos por flor se determinó contando bajo el microscopio estereoscópico el número de óvulos en un carpelo de 30 flores y se multiplicó por el número total de carpelos de cada flor (igura 1).

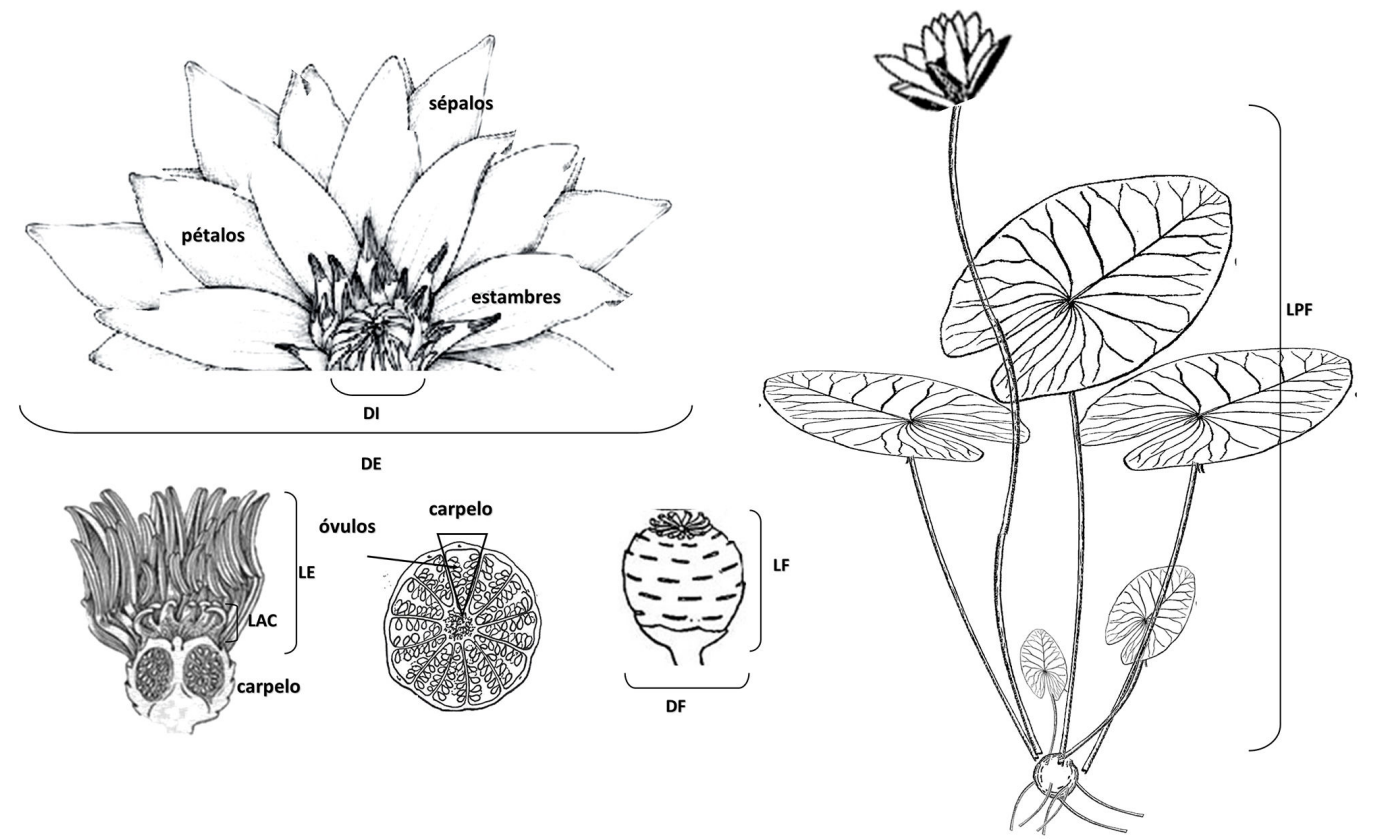

Figura 1. Características morfológicas evaluadas en Nymphaea gracilis. DE $=$ diámetro externo, DI $=$ diámetro interno, $\mathrm{LPF}=$ longitud del pedúnculo floral, $\mathrm{LE}=$ longitud de estambres, $\mathrm{LAC}=$ longitud de apéndices carpelares, $\mathrm{LF}=$ longitud de fruto, $\mathrm{DF}=$ diámetro de fruto. 
Fenología. Para determinar el tiempo promedio de vida de las flores y su comportamiento fenológico, 30 flores de distintas plantas distribuidas a lo largo del cuerpo de agua se siguieron durante la antesis. Como indicadores del estado de maduración floral se registró, la disponibilidad de polen (anteras dehiscentes o indehiscentes), receptividad estigmática, volumen de líquido estigmático, cierre definitivo (considerado solo después de un día sin exposición de las partes florales) y hundimiento de la flor (asociado al enrollamiento del pedúnculo floral y la retracción de la flor bajo el nivel del agua). Estos datos también permitieron determinar la sincronía temporal entre la expresión sexual masculina y femenina, ya sea simultánea (adicogamia) o desfasada (dicogamia). El estigma se consideró receptivo por la presencia evidente de líquido estigmático, una prueba simple de peróxido de hidrogeno al $40 \%$ al final de cada día permitió determinar la actividad de la peroxidasa en el estigma e inferir su receptividad a través del burbujeo al contacto con peróxido (Dafni et al. 2005). Adicionalmente, y durante los días que duró la antesis, cada flor se observó directamente a intervalos de media hora, esto con la intención de determinar la hora de apertura y cierre diario, así como el tiempo de exposición completa de las partes florales. Las diferencias diarias de apertura, cierre y exposición completa de las flores se exploraron mediante una ANOVA de un factor para medidas repetidas ejecutado en SPSS (IBM $\underline{2015)}$.

Para determinar si el volumen de líquido estigmático cambiaba a lo largo de los días de antesis y entre el inicio y cierre diario de la flor se aplicó una prueba seriada a 20 flores con antesis de primer, segundo y tercer día; a cada flor se le midió en dos ocasiones y durante el mismo día $(10: 00$ y 15:00 h) el volumen de líquido estigmático mediante una jeringa de $0.5 \mathrm{ml}$. Los datos se analizaron en conjunto mediante una ANOVA para medidas repetidas con el día $(1,2,3)$ y la hora $(10: 00$ y 15:00 h) como factores. Las diferencias en el volumen de líquido entre las dos horas evaluadas de cada día se probaron con una $t$-Student para muestras dependientes. Ambos análisis se ejecutaron en el programa SPSS (IBM 2015).

La producción de flores por planta y su apertura simultánea se evaluó en 12 plantas marcadas durante la época de crecimiento, en cada una se registró la fecha de apertura y cierre definitivo de la(s) flor(es) que produjo, además de la fecha de inicio y término de producción de polen y de receptividad del gineceo de cada flor. La sincronía entre las fases femeninas y/o masculinas de las flores de una misma planta y de plantas diferentes se definió como el número de días durante los cuales una flor estuvo receptiva y/o produjo polen paralelamente con otra flor, con esto se determinó en una hoja de cálculo el índice de sincronía $\left(x_{i}\right.$, Apéndice 1) para las flores de una planta $\mathrm{y}$ para las plantas de la población según la fórmula de Augspurger (1983).

Visitantes florales. Durante cuatro días consecutivos se observaron y capturaron los insectos que visitaron las flores de $N$. gracilis (16 h acumuladas de observación). La frecuencia de visitas de cada especie de insecto, así como el comportamiento y el contacto de la carga de polen con el estigma, se evaluó por observación directa durante 6 a 8 intervalos de $20 \mathrm{~min}$ por día y durante el tiempo que las flores estuvieron abiertas. Para determinar la región corporal y abundancia de polen transportado, de 5 a 10 insectos de cada especie se colectaron y colocaron en $2 \mathrm{ml}$ de glicerina al $50 \%$ y se mantuvieron en agitación constante para separar de su cuerpo los granos de polen. El número de granos de polen de $N$. gracilis por visitante se determinó como el promedio de los conteos de polen bajo el microscopio óptico de cuatro alícuotas multiplicado por el factor de dilución.

Para los visitantes más frecuentes, se comparó la frecuencia de visitas por horas mediante una prueba de $\chi^{2}$ en el programa SPSS (IBM 2015) y se calculó: 1) Índice de Probabilidad de Polinización (PPI), 2) Valor de Importancia de Polinización y 3) Índice de Importancia de Polinización $(P I I)$, las fórmulas de estos índices y sus referencias se muestran en la Apéndice 1.

Sistema reproductivo. A partir de la estimación de polen y óvulos por flor calculada en el apartado de morfometría, se calculó el sistema de apareamiento mediante la relación polen: óvulo (Cruden 1977, 2000). Adicionalmente se determinó la carga de polen sobre el estigma en 20 estigmas expuestos a polinización natural y colectados 24 horas después de la antesis. Los estigmas se lavaron con $3.0 \mathrm{ml}$ de agua y azul de anilina, los granos de polen y los tubos polínicos se contaron bajo el microscopio óptico en cinco alícuotas, el número de granos de polen en el estigma se determinó como el promedio de las cinco alícuotas multiplicadas por el factor de dilución (Kearns \& Inouye $\underline{1993})$.

Para medir la eficiencia reproductiva natural, en 40 frutos se calculó el número de semillas totales (completas y abortivas) y el valor resultante se multiplicó por el número total de lóculos por fruto. El número promedio de semillas abortadas por fruto dividido por el número promedio de semillas por fruto indica la proporción de semillas abortadas (Barrios \& Ramírez 2008).

Para determinar la biomasa asignada a cada uno de los verticilos florales y la inversión de biomasa masculina respecto a la femenina, 30 flores se separaron en las distintas partes florales y se secaron en un horno de convección a $30{ }^{\circ} \mathrm{C}$ por cinco días o hasta peso contante. El peso seco de cada uno de los verticilos se sumó para determinar la biomasa total de cada flor, adicionalmente y 
de acuerdo con Ramsey (1993), se calcularon cuatro índices que reflejan la asignación de recursos: 1) relación biomasa reproductiva (androceo y gineceo): biomasa vegetativa (cáliz y corola), 2) relación biomasa androceo: biomasa gineceo, 3) biomasa masculina relativa (M1, Apéndice 1) y 4) biomasa masculina post-cigótica (M2, Apéndice 1).

La biomasa de frutos y semillas se calculó utilizando 40 frutos completamente desarrollados, los cuales se midieron a lo largo y ancho con la ayuda de un vernier digital (Figura 1) y se secaron a $40{ }^{\circ} \mathrm{C}$ por tres semanas o hasta peso contante, cada fracción se pesó por separado. Esto permitió el cálculo del costo relativo de inversión materna (pericarpo): progenie (semillas) de acuerdo con Barrios \& Ramírez (2008) (Apéndice 1) y del cambio que ocurre desde la condición floral a la fase final de frutos (relación peso fruto: peso flor).

Tratamientos de polinización. Se aplicaron 5 tratamientos de exclusión de las flores de $N$. gracilis a través del aislamiento de los botones de diferentes plantas con bolsas de polietileno perforadas para evitar la entrada de insectos. Los tratamientos fueron: 1) Autogamia: 20 botones se aislaron al inicio de la antesis y se mantuvieron $\sin$ manipulación, 2) Agamospermia: 20 botones se emascularon al inicio de la antesis y se aislaron, 3) Geitonogamia: 10 botones se aislaron después de que fueron emasculados y polinizados el primer día con polen de flores de la misma planta, 4) Xenogamia natural: 20 botones se emascularon el primer día de antesis y se mantuvieron sin aislamiento, 5) Xenogamia manual y receptividad del estigma, para estos tratamientos 60 botones florales se aislaron al inicio de la antesis y se polinizaron con una mezcla de polen de flores de otras plantas en los diferentes días de la antesis: 20 botones se polinizaron el primer día de antesis, 10 el segundo, tercer, cuarto y quinto día de antesis respectivamente. 6) Control (polinización abierta): 10 flores de primer día se marcaron y mantuvieron sin manipulación durante todo el experimento.

Todas las flores se mantuvieron aisladas hasta la producción de frutos y semillas, y se recolectaron tres semanas después del inicio de la antesis. Para cada tratamiento se contó el número semillas producidas (completas y abortadas) y óvulos abortados. El número de óvulos por prueba se determinó multiplicando el número de flores bajo experimentación por el promedio de óvulos por flor. La estrategia reproductiva de la especie se determinó utilizando los siguientes índices: 1) Tasa de fecundidad cruzada (IFC) para frutos y semillas, 2) Índice de incompatibilidad (ISI), 3) Índice de autogamia (IAS) y 4) Éxito reproductivo relativo (ERR), las fórmulas y referencias de cada índice se indican en la Apéndice 1.

La producción de frutos y semillas entre tratamientos se comparó mediante una prueba G (Sokal \& Rohlf 2012) ejecutada en el paquete PROC FREC de SAS (2013), esto considerando la distribución de frecuencias de frutos y semillas producidos y no producidos con relación al total de flores entre pruebas experimentales.

La limitación reproductiva por polinizadores se calculó como la razón de semillas producidas por polinización abierta respecto a las producidas por xenogamia manual (Rivera-Hutinel \& Acevedo-Orellana 2017).

Depresión endogámica: El cálculo del rendimiento relativo de frutos y semillas (RP, Apéndice 1) de las pruebas de autogamia y xenogamia manual se usó como medida de depresión endogámica según Ågren \& Schemske (1993).

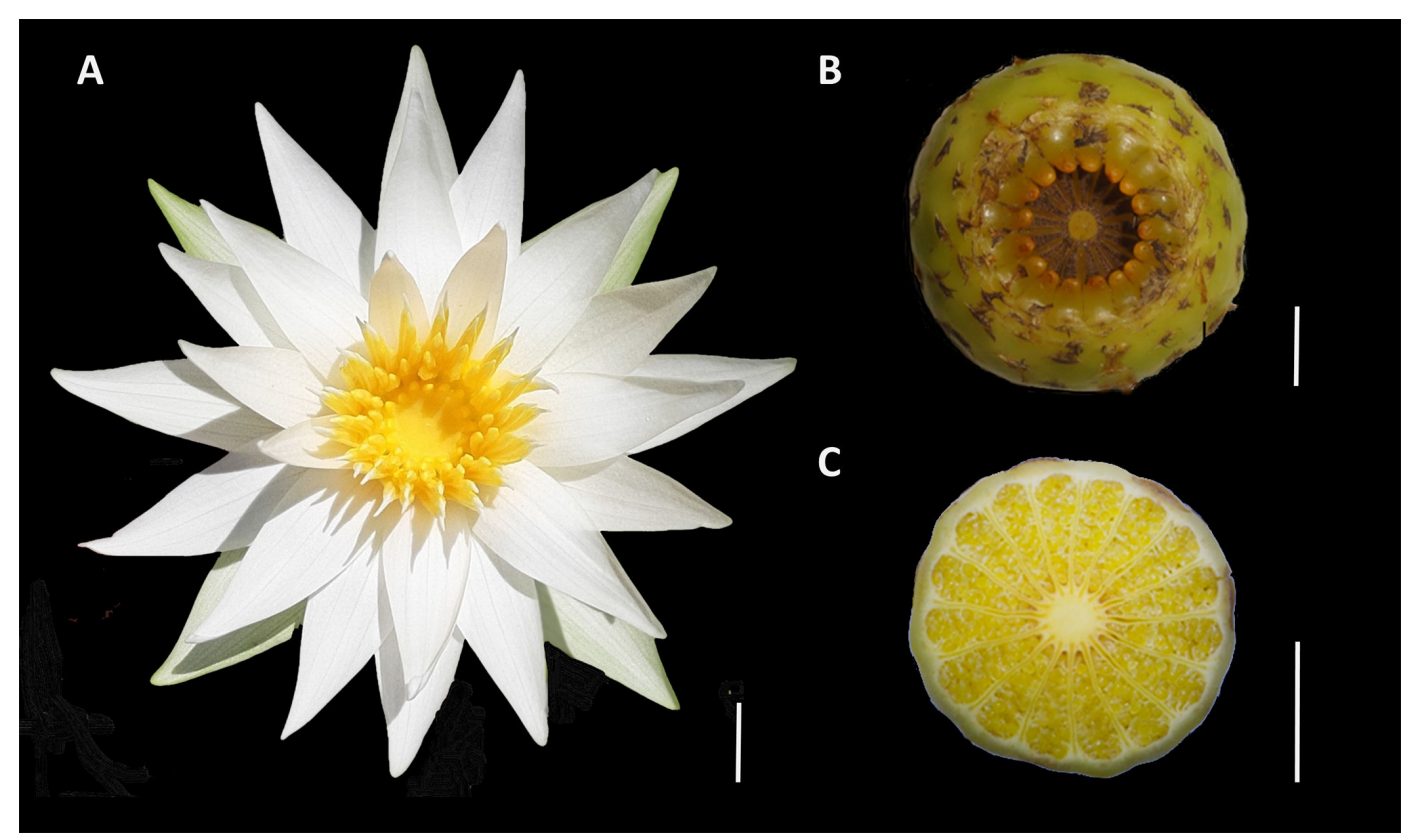

Figura 2. Flores (A), frutos (B) y sección transversal de carpelos (C) de Nymphaea gracilis. Las barras blancas equivalen a un centímetro de longitud. 


\section{Resultados}

Morfometría y producción de polen y óvulos. Nymphaea gracilis posee flores solitarias, vistosas, hermafroditas y de antesis diurna (Figura 2A). Sus dimensiones son: diámetro externo $6.0-12.3 \mathrm{~cm}(\overline{\mathrm{x}}=9.0 \pm 1.5 \mathrm{~cm})$; diámetro interno $0.7-1.4 \mathrm{~cm}(\overline{\mathrm{x}}=1.0 \pm 0.1 \mathrm{~cm})$ y longitud de pedúnculo floral $37.4-70.8 \mathrm{~cm}(\overline{\mathrm{x}}=54.7 \pm 9.3 \mathrm{~cm})$ con $8.0-28.0 \mathrm{~cm}(\overline{\mathrm{x}}=19.7$ $\pm 4.7 \mathrm{~cm})$ sobre el nivel del agua. El perianto es actinomorfo y diclamídeo, está compuesto por cuatro sépalos lanceolados blanco-verdosos al interior y verdes con manchas lineares obscuras en el exterior; adicionalmente presenta $15-26(\overline{\mathrm{x}}=19 \pm 12.5)$ pétalos blancos, lanceolados y contrastantes con los 37-78 ( $\overline{\mathrm{x}}=58 \pm 11)$ estambres lineares que se disponen en varias series de diferentes tamaños. Los estambres más cercanos al disco estigmático miden $0.5-1.2 \mathrm{~cm}$ de longitud $(\overline{\mathrm{x}}=0.9 \pm 0.2 \mathrm{~cm}$ de longitud), están rodeados por dos series más de estambres, una intermedia de 1.3-2.2 cm longitud $(\bar{x}=1.7 \pm 0.18 \mathrm{~cm}$ de longitud) y una externa de $2.3-3.5 \mathrm{~cm}$ de longitud $(\overline{\mathrm{x}}=2.7$ $\pm 0.3 \mathrm{~cm}$ de longitud). Los estambres intermedios $\mathrm{y}$ externos tienen el ápice de conectivo muy desarrollado y producen significativamente más granos de polen que los estambres internos $\left(F_{4,87}=18.7, P<0.0001\right.$, Tabla 1$)$. Todas las anteras son de dehiscencia longitudinal e introrsa. Una flor puede producir en promedio $58 \pm 10$ anteras y 547,844 $\pm 55,117$ granos de polen (Tabla 1).

Tabla 1. Promedio ( $\pm \mathrm{DE}$ ) del número de anteras, polen, óvulos y relación polen:óvulos de Nymphaea gracilis.

\begin{tabular}{lc}
\hline Estructura & $\overline{\mathbf{x}} \pm \mathbf{D E}$ \\
\hline Anteras internas & $23 \pm 5$ \\
Granos de polen por antera interna & $3,905 \pm 1,839 \mathrm{a}$ \\
Anteras intermedias & $19 \pm 5$ \\
Granos de polen por antera intermedia & $10,521 \pm 8,389 \mathrm{~b}$ \\
Anteras exteriores & $16 \pm 4$ \\
Granos de polen por antera externa & $16,133 \pm 10,313 \mathrm{~b}$ \\
Anteras totales por flor & $58 \pm 10$ \\
Granos de polen totales por flor & $547,844 \pm 55,117$ \\
Carpelos por flor & $15 \pm 2$ \\
Óvulos por carpelo & $79 \pm 35$ \\
Óvulos totales por flor & $1,207 \pm 613$ \\
Relación polen: óvulos & $454: 1$ \\
\hline
\end{tabular}

Diferentes letras indican diferencias significativas usando una prueba de ANOVA con una significancia de $P<0.0001$.
El gineceo es apocárpico, está constituido por 11-19 $(\overline{\mathrm{x}}=15 \pm 2)$ carpelos lateralmente conniventes $\mathrm{y}$ con apéndices carpelares cónicos de 2-3.1 mm de largo, en conjunto forman un disco estigmático radiado de 0.4$1.5 \mathrm{~cm}^{2}$ de superficie $\left(\overline{\mathrm{x}}=0.86 \pm 0.28 \mathrm{~cm}^{2}\right)$, cóncavo y de color amarillo en el $80 \%$ de la población (Figura 2A, C), el $20 \%$ restante mostró estigmas de color rojo o purpura intenso. Desde el eje central hasta la base de los apéndices carpelares, la superficie estigmática está cubierta de papilas multicelulares que forman una amplia zona receptora de polen. El disco estigmático y los pétalos se tiñeron de rojo con la técnica de rojo neutro, indicando el sitio de emisión de olor el cual es muy ligero y afrutado. Una flor puede producir 476-2,856 ( $\overline{\mathrm{x}}=1,207 \pm 613$, Tabla 1$)$ óvulos anátropos con placentación laminar, rodeados por mucílago transparente y persistente en el fruto.

Fenología. Las flores de $N$. gracilis son protóginas y mostraron una longevidad de 4-6 días $(\overline{\mathrm{x}}=4.5 \pm 0.5)$, máxima apertura floral diaria de $6 \mathrm{~h}$ y mínima de $3 \mathrm{~h}$ $(\overline{\mathrm{x}}=4.6 \pm 0.5)$, iniciando desde las 10:00 $\mathrm{h}$ y terminando hacia las 17:00 h (igura 3). El tiempo de exposición diaria de las flores varia a lo largo de la vida de la flor, las flores de primer y segundo día exponen sus flores por $4.3 \pm 0.6 \mathrm{~h}$ y $4.2 \pm 0.6 \mathrm{~h}$ respectivamente, mientras que las flores de los días subsiguientes las exponen por 50-60 min más $\left(F_{4,22}=15.5, P<0.0001\right.$, Figura 3).

La mayor receptividad estigmática se presentó en el primer y segundo día de la antesis, cuando aún estuvo presente el líquido estigmático, sin embargo, la reacción del peróxido aún se consideró positiva durante las primeras horas del tercer día ya que los estigmas produjeron un pequeño burbujeo. Las comparaciones múltiples indican que el volumen de líquido estigmático difiere entre los tres primeros días de antesis $\left(F_{2,18}=240, P<0.0001\right)$ y entres las horas del día $\left(F_{1,19}=23.9, P<0.0001\right)$.

El primer día de la antesis las flores emitieron un suave aroma afrutado, al principio se mantuvieron con sus estructuras florales parcialmente extendidas formando una copa que solo dejó expuesto al estigma. En este momento el $100 \%$ de los estigmas (Figura 4) estuvo cubierto por líquido estigmático y se consideró receptivo al polen. Una flor de primer día llegó a contener hasta $0.4 \mathrm{ml}$ de líquido estigmático $(\overline{\mathrm{x}}=0.23 \pm 0.08 \mathrm{ml})$, sin embargo, su volumen disminuyó a lo largo del día y antes del cierre floral los estigmas solo conservaron un poco más de la mitad del líquido estigmático inicial $(\overline{\mathrm{x}}=0.16 \pm 0.05 \mathrm{ml}$; $\left.t_{19}=13.3, P<0.0001\right)$.

En el inicio del segundo día, los estigmas mantuvieron cerca de la tercera parte del líquido estigmático inicial $(\overline{\mathrm{x}}=0.08 \pm 0.03 \mathrm{ml})$ por lo que aún se consideraron receptivos (Figura 4). Sin embargo, el volumen de líquido 


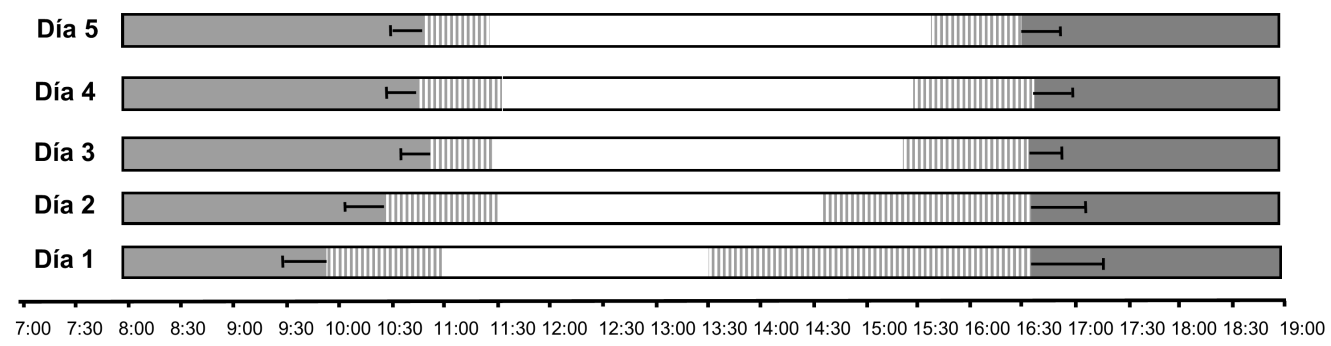

Tiempo (Horas)

Figura 3. Tiempos de apertura de las flores de Nymphaea gracilis durante cinco días de antesis. Las barras indican el tiempo promedio de apertura y cierre $( \pm \mathrm{DE})$. Las barras ralladas representan el tiempo de inicio de apertura y cierre de las flores, las barras blancas flores abiertas y las barras obscuras flores completamente cerradas.

continúo disminuyendo y en comparación con el inicio del segundo día, antes del cierre floral los estigmas solo se mantuvieron ligeramente húmedos $(\overline{\mathrm{x}}=0.04 \pm 0.01 \mathrm{ml}$; $\left.t_{19}=12.3, P<0.0001\right)$ y el aroma floral se hizo casi imperceptible. Al mismo tiempo, en el $66 \%$ de las flores los estambres más externos y grandes iniciaron su dehiscencia y liberación de polen (Figura 4), de forma que la expresión sexual masculina y femenina ocurrió simultáneamente desde el final de este día y podría considerarse como una protoginia incompleta.

En el tercer día, el estigma menos brillante y sin líquido estigmático o en algunos casos (26\%) solo humedecido durante las primeras horas del día $(\overline{\mathrm{x}}=0.02 \pm 0.02 \mathrm{ml})$, se mantuvo cubierto por las anteras más próximas y aún indehiscentes, mientras que los estambres más externos terminaron de abrir (Figura 4). En el cuarto y quinto día todas las partes florales se expandieron completamente y dejaron expuesto al estigma ya sin líquido estigmático y por lo tanto no receptivo (Figura 4); las anteras abrieron progresiva y centrípetamente de forma que al final del quinto día, los estambres internos y más próximos al estigma fueron los últimos en completar su dehiscencia (Figura 4).

Los patrones de apertura, cierre y exposición de las flores mostraron variaciones a lo largo de la antesis (Figura 3). Las flores de primer día inician su apertura desde las 10:00 am $(\overline{\mathrm{x}}=10: 33 \pm 0: 22 \mathrm{~h}), 40-45$ min más temprano que las flores de segundo, tercer, cuarto y quinto día $\left(F_{4,22}=17.2\right.$, $P<0.0001)$. Después de iniciada la apertura, las flores de primer día se mantuvieron parcialmente abiertas por más de una hora $(\overline{\mathrm{x}}=72 \pm 15 \mathrm{~min})$ antes de exponer completamente todas sus partes florales, mientras que las flores de segundo y tercer día tardaron de 39-48 min y las de cuarto y quinto día $30 \min \left(F_{4,22}=77.9, P<0.0001\right)$. La exposición completa de las flores de primer día duró $3.1 \pm 0.57 \mathrm{~h}$, es decir cerca de 20 min menos que las flores de segundo día $(\overline{\mathrm{x}}=3.4 \pm 0.77 \mathrm{~h})$, y de 60 a $70 \mathrm{~min}$ menos que las flores de los días subsiguientes $\left(F_{4,22}=40.5, P<0.0001\right)$. Además, las flores de primer día iniciaron su cierre $35 \mathrm{~min}$ antes que las flores de segundo día y de 70-75 min antes que las flores de tercer, cuarto y quinto día $\left(F_{4,23}=35.8, P<0.0001\right.$, Figura 3).

घ Anteras internas dehiscentes $\square$ Anteras intermedias dehiscentes $\square$ Anteras externas dehiscentes $\square$ Estigma receptivo

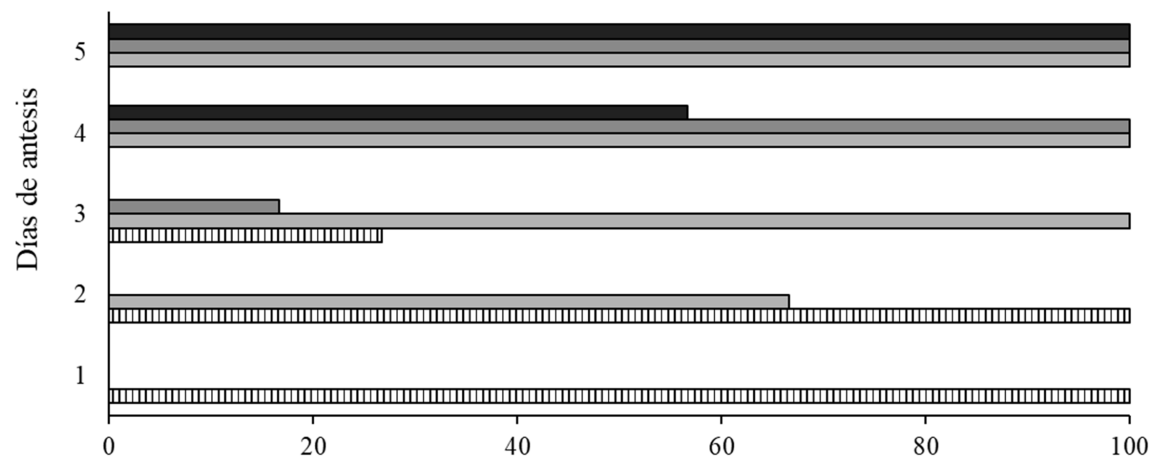

Porcentaje de flores (\%)

Figura 4. Porcentaje de flores con estigmas receptivos y dehiscencia de anteras de las flores de Nymphaea gracilis durante cinco días de antesis. 
Seis días después del primer día de antesis las flores no abrieron y tendieron a sumergirse por el enrollamiento del pedúnculo, permanecieron sumergidas evitando su depredación por aves acuáticas como Fulica americana y madurando por cuatro semanas. El fruto maduro es una baya de color verde claro y cubierto por las cicatrices de los sépalos, pétalos $\mathrm{y}$ estambres que se desintegran progresivamente (Figura 2B), mide de $1.5-2.7 \mathrm{~cm}$ de largo $(\overline{\mathrm{x}}=2.0 \pm 0.3)$ y $1.7-3.0 \mathrm{~cm}$ de diámetro $(\overline{\mathrm{x}}=2.3 \pm 0.4 \mathrm{~cm})$; la dehiscencia fue irregular y dejó libres las semillas que flotaron por algún tiempo gracias a la presencia de un arilo translúcido que las rodea parcialmente. Las semillas son esféricas, verde grisáceas y de 0.9-1.7 $\mathrm{mm}$ de diámetro $(\overline{\mathrm{x}}=1.4 \pm 0.3 \mathrm{~mm})$. Transcurrieron cerca de 5 semanas desde la polinización hasta dispersión de las semillas.

Las plantas se desarrollaron desde finales de agosto hasta mediados de noviembre, durante esta temporada formaron $5.1 \pm 1.2$ flores, el índice de sincronía $\left(x_{i}\right)$ entre la fase masculina y femenina promedio de la planta fue de $0.34 \pm 0.25$ y el índice de floración sincrónica para la población de $0.6 \pm 0.1$, lo que significa que las plantas presentaron una superposición parcial en la expresión sexual de sus flores o dicogamia incompleta.
Visitantes florales. Las flores de $N$. gracilis fueron visitadas por insectos de los órdenes Hymenoptera, Diptera y rara vez alguna especie de Coleoptera y Hemiptera (Tabla 2), cada uno con diferentes estrategias de forrajeo. Apis mellifera fue el principal visitante floral (VR $=60 \%$ ), el que transportó el mayor porcentaje de polen ( $\mathrm{PCP}=72 \%$ ) y en el que todos los individuos trasportaron polen de $N$. gracilis $(\mathrm{P} B \mathrm{P}=100 \%)$, por lo que se considera el visitante que mayor probabilidad tiene de ser un polinizador importante de $N$. gracilis $(\mathrm{PPI}=72 \%$, Tabla 2). Este himenóptero visitó en promedio 15 flores/h y colectó vigorosamente polen, el cual transportó en el abdomen y patas, su mayor actividad fue entre las 10:00 y 13:00 h cuando pasó rápidamente de una flor a otra (Figura 5). En las flores de primer día algunas abejas aterrizaron en el estigma o cerca de él, pero en general permanecieron poco tiempo en ellas, mientras que en las flores de segundo, tercer, cuarto y quinto día se mantuvieron por más tiempo debido a la presencia de polen. El índice $P \mathrm{II}=99 \%$ indica que A. mellifera realizó la mayor contribución a la polinización de $N$. gracilis.

El díptero Allograpta obliqua fue el segundo insecto que más frecuentemente visitó las flores de $N$. gracilis

Tabla 2. Visitantes florales de Nymphaea gracilis. Carga de polen, Índice de probabilidad de polinización (PPI) e Índice de importancia de polinización $(P \mathrm{II}) . \mathrm{PCP}=$ Proporción media de polen presente en los insectos, $\mathrm{P} B \mathrm{P}=$ Proporción de individuos que trasportaban polen, $\mathrm{PE}=$ Eficiencia básica de polinización, $\mathrm{VR}=$ Tasa de visitas, $\mathrm{C}=$ Constancia, $\mathrm{PCC}=$ Capacidad de trasporte de polen, $\mathrm{PIV}=\mathrm{Valor}$ de importancia de polinización. $\mathrm{A}=$ abdomen, $\mathrm{T}=$ tórax, $\mathrm{P}=$ patas, $\mathrm{SP}=\sin$ polen.

\begin{tabular}{|c|c|c|c|c|c|c|c|c|c|c|c|c|c|}
\hline \multirow{2}{*}{$\begin{array}{l}\text { Orden y } \\
\text { Familia }\end{array}$} & \multirow{2}{*}{ Especie } & \multirow{2}{*}{$\begin{array}{c}\text { Visitas } \\
\text { (individuos/hr) }\end{array}$} & \multirow{2}{*}{$\begin{array}{c}\text { Sitio de } \\
\text { transporte }\end{array}$} & \multicolumn{2}{|c|}{$\begin{array}{l}\text { Número de granos de polen } \\
\qquad(\overline{\mathbf{x}} \pm \mathrm{DE})\end{array}$} & \multicolumn{3}{|c|}{$\begin{array}{l}\text { Probabilidad de } \\
\text { polinización }\end{array}$} & \multicolumn{5}{|c|}{ Importancia de polinización } \\
\hline & & & & Totales & $\begin{array}{c}\text { Nymphaea } \\
\text { gracilis }\end{array}$ & $\mathbf{P C P}$ & $\mathbf{P B P}$ & PPI & $\mathbf{P E}$ & $\mathbf{V R}$ & PCC & PIV & $\begin{array}{l}\text { PII } \\
(\%)\end{array}$ \\
\hline \multicolumn{14}{|l|}{ Hymenoptera } \\
\hline Apidae & Apis mellifera & 14.9 & $\mathrm{~A}, \mathrm{P}, \mathrm{T}$ & $\begin{array}{c}11,596 \pm \\
3,372\end{array}$ & $8,386 \pm 4,451$ & 0.723 & 1 & 0.723 & 0.84 & 0.60 & 0.633 & 0.228 & 99 \\
\hline Megachilidae & $\begin{array}{l}\text { Chelostoma sp. } \\
\text { Ceratina sp. }\end{array}$ & $\begin{array}{c}1 \\
0.5\end{array}$ & $\begin{array}{l}\mathrm{P} \\
\mathrm{SP}\end{array}$ & & & & & & & & & & \\
\hline \multicolumn{14}{|l|}{ Diptera } \\
\hline \multirow[t]{3}{*}{ Syrphidae } & Allograpta obliqua & 5.8 & A & $682 \pm 282$ & $2 \pm 1.0$ & 0.040 & 0.7 & 0.028 & 0.78 & 0.23 & 0.002 & $1.5^{-5}$ & 0.006 \\
\hline & Asemosyrphus sp. & 1 & $\mathrm{P}, \mathrm{T}$ & $512 \pm 293$ & $9 \pm 5$ & 0.018 & 0.6 & 0.011 & 0.025 & 0.04 & 0.0006 & $1.9^{-7}$ & $8.5^{-5}$ \\
\hline & Platycherius sp. & 1.2 & A & $458 \pm 284$ & $4 \pm 2$ & 0.004 & 0.2 & 0.001 & 0.48 & 0.03 & 0.0001 & $7.4^{-9}$ & $3.2^{-6}$ \\
\hline Tabanidae & Sp. 1 & 0.2 & SP & - & - & - & - & - & - & - & - & - & - \\
\hline \multicolumn{14}{|l|}{ Coleoptera } \\
\hline Cantharidae & Sp. 1 & 0.5 & SP & - & - & - & - & - & - & - & - & - & - \\
\hline \multicolumn{14}{|l|}{ Hemiptera } \\
\hline Pentatomide & Arma sp. & 0.2 & SP & - & - & - & - & - & - & - & - & - & - \\
\hline
\end{tabular}




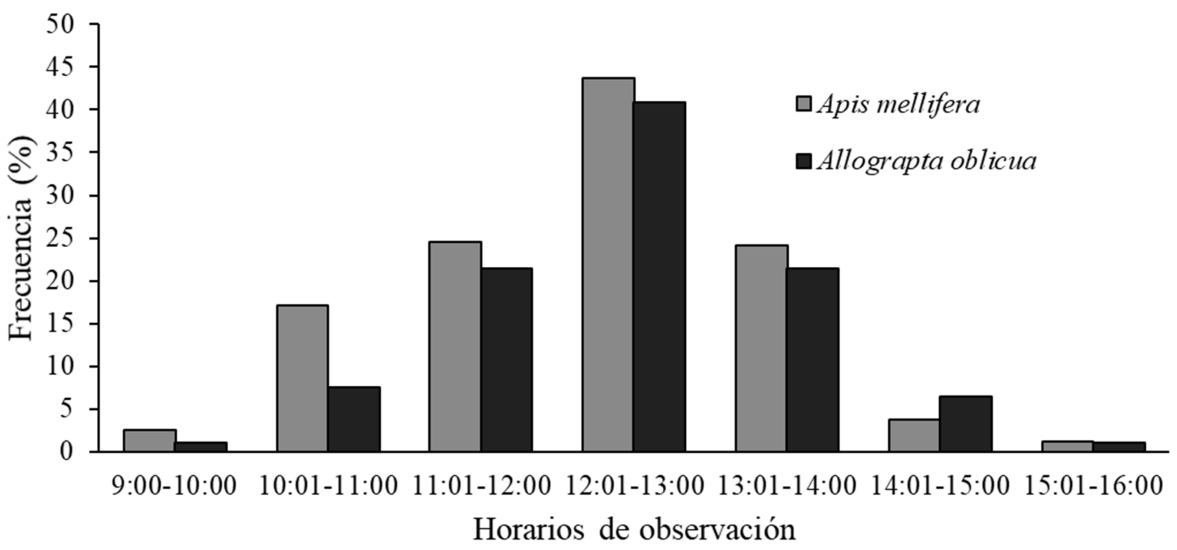

Figura 5. Frecuencia de visitas de Apis mellifera y Allograpta oblicua en flores de Nymphaea gracilis.

(VR $=23 \%$, Tabla 2) la frecuencia de visitas fue similar a la de las abejas melíferas $\left(\chi_{6}^{2}=8.13, P>0.05\right.$, Figura 5 $)$, sin embargo, una de cada tres flores observadas presentó a estos dípteros y a los del género Asemosyrphus (Lejops) ahogados en el líquido estigmático pocas horas después de la apertura floral. Allograpta oblicua y Asemosyrphus sp. transportaron un porcentaje muy bajo de polen de N. gracilis ( $\mathrm{PCP}=4$ y $1 \%$ respectivamente, Tabla 2$)$, y solo el 70 y $60 \%$ de los visitantes de cada una de estas especies lo transportaban, lo que hace que el valor PPI de cada especie de insectos sea bajo (2\% y $1 \%$ respectivamente, Tabla 2). Allogapta oblicua obtuvo los valores más altos de PE porque visitó un mayor número de flores, sin embargo, su contribución a la polinización de la especie $(P I I=0.006 \%)$ es insignificante.

El resto de los insectos no presentaron polen o fue escaso (Tabla 2) y sus visitas a las flores fueron breves como en Chelostoma sp. o muy prolongadas como en los hemípteros y coleópteros, los cuales se posaron principalmente sobre partes florales no reproductivas. Ningún insecto se observó bebiendo el líquido estigmático.

Sistema reproductivo. Las flores presentaron en promedio $547,844 \pm 55,117$ granos de polen por flor (Tabla 1). En contraste, la cantidad de óvulos por flor fue más de 400 órdenes de magnitud menor $(\overline{\mathrm{x}}=1,207 \pm 613$, Tabla 1 $)$. La relación polen: óvulo estimada fue de 454:1 (Tabla 1), que corresponde a la categoría de xenogamia facultativa de acuerdo con la clasificación de Cruden (1977). Es decir, el sistema reproductivo de $N$. gracilis es preponderantemente por fecundación cruzada y sin mecanismos para evitar la autofecundación.

El número promedio de granos de polen encontrados sobre un grupo de estigmas con $0.6 \pm 0.1 \mathrm{~cm}^{2}$ del segundo día de antesis fue de $650 \pm 378$ y un porcentaje de polen germinado del $22 \%$. Los granos de polen sobre el estigma representaron 0.6 veces el número de óvulos por flor, el $0.001 \%$ de los granos de polen por flor y el $0.002 \%$ de los granos de polen por anteras externas. La eficiencia reproductiva natural fue de 1,093 \pm 547 semillas por fruto, es decir cerca del $90 \%$ de los óvulos producidos de manera natural por una flor se fecundaron. La fracción de semillas abortadas respecto a las semillas totales fue de $71 \pm 26$ semillas $(6.5 \%)$.

La relación biomasa reproductiva: biomasa vegetativa fue de 1.2:1 g, lo que indica que en las flores hay mayor inversión sobre las estructuras reproductivas (Tabla 3). De la biomasa asignada a estructuras reproductivas, la mayor cantidad se destinó al androceo $(\mathrm{M} 1=0.57)$, resultando en una relación biomasa androceo: gineceo de 1.4:1 g, mientras que el cálculo de la masa reproductiva post-cigótica $(\mathrm{M} 2=0.05)$ indica una mayor asignación a la biomasa femenina después de la fecundación.

Tabla 3. Biomasa $(\overline{\mathrm{x}} \pm \mathrm{DE})$ de verticilos florales $(\mathrm{n}=30)$ y frutos $(\mathrm{n}=40)$ de Nymphaea gracilis.

\begin{tabular}{lcc}
\hline & Peso seco $(\mathbf{m g}) \overline{\mathbf{x}} \pm$ DE & Porcentaje $(\%)$ \\
\hline Cáliz & $75.15 \pm 43.06$ & 22.45 \\
Corola & $79.00 \pm 42.66$ & 23.60 \\
Androceo & $103.78 \pm 44.10$ & 31.00 \\
Gineceo & $76.84 \pm 42.13$ & 21.95 \\
Flor completa & $334.75 \pm 171.95$ & 100 \\
Pericarpio & $665.54 \pm 366.01$ & 24.35 \\
Semillas & $2,076.00 \pm 1,417.55$ & 75.65 \\
Fruto completo & $2,741.54 \pm 1,783.56$ & 100 \\
\hline
\end{tabular}

Los frutos de $N$. gracilis presentaron un peso seco promedio de $2.7 \mathrm{~g}$ (Tabla 3), de los cuales el $75.65 \%$ corresponde a semillas y $24.35 \%$ al pericarpio, en consecuencia, la relación pericarpio: semillas fue de $0.32 \mathrm{~g}$, indicando que existe una mayor inversión de biomasa en la producción de semillas que en la producción de pericarpio. La relación peso fruto: peso flor $(8.419 \mathrm{~g})$ indica un incremento de biomasa de un poco más de 8 veces desde la fase de flor hasta la formación de frutos y semillas. 
Tratamientos de polinización. Los índices calculados indican que las plantas de $N$. gracilis son autocompatibles $(\mathrm{ISI}=1.0)$ y con autogamia parcial (IAS $=0.88)$. La tasa de fecundidad cruzada muestra un sistema de cruzamiento mixto tanto a nivel de frutos $\left(\mathrm{IFC}_{\text {fruto }}=0.44\right)$ como de semillas $\left(\mathrm{IFC}_{\text {semilla }}=0.48\right)$. La razón de las semillas producidas por polinización abierta respecto a las producidas por xenogamia manual (0.4), sugiere limitación de polen mediada por polinizadores.

La especie no es agamosperma. En la autogamia, geitonogamia, xenogamia y control el $70 \%$ o más de las flores formaron frutos sin que en alguno de ellos se presentara una proporción significativamente mayor. No obstante, la proporción de semillas producidas fue significativamente diferente entre los tratamientos; el tratamiento de geitonogamia produjo una mayor proporción de semillas que los tratamientos de autogamia $(G=2,188$, $P<0.0001)$, xenogamia manual $(G=3,699, P<0.0001)$, xenogamia natural $(G=8,325, P<0.0001)$ y control ( $G=1,241, P<0.0001)$; la autogamia fue el segundo mecanismo que produjo significativamente más semillas (Tabla 4). La fecundación manual mostró una disminución en la proporción de frutos a lo largo de la vida de la flor. Para el caso de las semillas, la mayor proporción se observó cuando las flores se fecundaron en el segundo día y fue significativamente mayor que las fecundaciones del primer $(G=1,234, P<0.0001)$, cuarto $(G=4,316, P<0.0001)$ y quinto día $(G=4,461, P<0.0001$, Tabla 4). El cálculo del ERR mostró mayor éxito reproductivo en los tratamientos de geitonogamia y autogamia, así como en la fecundación de flores en los primeros dos días de la antesis (Tabla 4).
Depresión endogámica. El cálculo del rendimiento relativo de frutos y semillas indicó que la depresión endogámica fue baja para el conjunto de frutos $(\mathrm{RP}=-0.1)$ y relativamente baja para semillas $(\mathrm{RP}=0.26)$.

\section{Discusión}

Morfometría y fenología Las características florales de la población estudiada de $N$. gracilis son consistentes con los reportes para otras poblaciones de México (Novelo \& Bonilla-Barbosa 1999, Calderón de Rzedowski \& Rzedowski 2010, Zepeda-Gómez 2017, Sandoval-Ortega 2020). Los análisis de su fenología floral muestran que es una especie diurna, con antesis de cinco días en promedio, receptividad estigmática los dos primeros días y protoginia incompleta por la liberación de polen desde el segundo día de floración.

En angiospermas basales, como Nymphaeaceae, el patrón más común son las flores protóginas con una antesis de dos días (Endress 2010) por ejemplo, Nymphaea mexicana Zucc. (Capperino \& Schneider 1985), N. odorata Aiton (Schneider \& Chaney 1981, Wiersema 1988), N. elegans Hook. (Schneider 1982), N. capensis Thunb. (Orban \& Bouharmont 1995) y $N$. naucali Burm.f. (Begum et al. 2010) son especies diurnas que exponen sus flores durante dos o tres días y al mantener sus estigmas receptivos durante el primer día, incrementan el éxito reproductivo femenino (Çetİnbaş \& Ünal 2014).

Ampliar la duración de la antesis, como se encontró en este estudio para $N$. gracilis, tiene implicaciones ecológicas importantes porque aumenta la exposición de los órganos

Tabla 4. Número de frutos, semillas y éxito reproductivo relativo (ERR) obtenidos de las pruebas de polinización en Nymphaea gracilis.

\begin{tabular}{|c|c|c|c|c|c|c|c|}
\hline & \multirow{2}{*}{ Botones iniciales } & \multicolumn{2}{|l|}{ Frutos } & \multicolumn{3}{|c|}{ Semillas } & \multirow{2}{*}{ ERR \% } \\
\hline & & Producidos & $\%$ & Esperadas & Producidas & $\%$ & \\
\hline Agamospermia & 20 & 0 & 0 & 24,140 & 0 & 0 & 0 \\
\hline Autogamia & 20 & 18 & 90 & 24,140 & $7,623 a$ & 32 & 28.4 \\
\hline Geitonogamia & 10 & 8 & 80 & 12,070 & $5,564 b$ & 46 & 36.8 \\
\hline Xenogamia natural & 20 & 14 & 70 & 24,140 & $2,330 \mathrm{c}$ & 10 & 6.8 \\
\hline Control & 10 & 8 & 80 & 12,070 & $2,430 \mathrm{~d}$ & 20 & 20.1 \\
\hline \multicolumn{8}{|l|}{ Xenogamia manual } \\
\hline Fecundación 1er día & 20 & 14 & 70 & 24,140 & $5,924 \mathrm{a}$ & 25 & 17.1 \\
\hline Fecundación $2^{\circ}$ día & 10 & 10 & 100 & 12,070 & $5,168 b$ & 43 & 42.8 \\
\hline Fecundación 3er día & 10 & 6 & 60 & 12,070 & $3,010 \mathrm{a}$ & 25 & 15.0 \\
\hline Fecundación $4^{\circ}$ día & 10 & 4 & 40 & 12,070 & $136 \mathrm{c}$ & 1 & 0.5 \\
\hline Fecundación $5^{\circ}$ día & 10 & 1 & 10 & 12,070 & $10 \mathrm{~d}$ & 0 & 0 \\
\hline
\end{tabular}

Diferentes letras indican diferencias significativas usando una prueba de $G$ con una significancia de $P<0.001$. 
reproductivos y el acceso de los polinizadores a ellos (Prance \& Anderson 1976, van der Velde 1986). En N. alba L. y N. candida C.Presl la extensión de la fase masculina en la antesis se ha interpretado como una mayor especialización en la biología floral ya que beneficia la atracción de un mayor número de polinizadores y de sus servicios (van der Velde 1986). Bajo esta consideración, la antesis de cinco días de $N$. gracilis y los horarios del despliegue floral de la fase masculina sugieren inicialmente una mayor exposición a favor de la fecundación cruzada. $\mathrm{Si}$ una flor de $N$. gracilis se mantiene abierta cinco días, las anteras liberarán polen durante cuatro de ellos, así los polinizadores tendrán $80 \%$ de probabilidades de acceder a flores funcionalmente masculinas, es decir $30 \%$ más que aquellas flores con antesis de 2 días. El despliegue floral diario podría contribuir en el mismo sentido, en $N$. gracilis se encontró que cada fase sexual tiene patrones de apertura y cierre particulares, por ejemplo, las flores funcionalmente masculinas tardan menos tiempo en abrir y se exponen por más tiempo que las flores funcionalmente femeninas. En otras especies de Nymphaea el patrón de apertura floral también muestra variaciones diarias y parece estar relacionado con la intensidad de la luz solar, pero sobre todo con la temperatura ambiental (Prance \& Anderson 1976, Wiersema 1988, Hirthe \& Porembski 2003, Begum et al. 2010).

Extender la fase femenina también podría ser una estrategia para aumentar las probabilidades de contacto con polinizadores potenciales. En la población de estudio la función femenina se alargó hasta el segundo día de la antesis, es decir el estigma estuvo receptivo el doble de lo que típicamente se reporta para las especies del género (Wiersema 1988), aumentando así de 20 a $40 \%$ las oportunidades de que un polinizador la visite.

Las pruebas de polinización cruzada artificial confirmaron que la receptividad del estigma en $N$. gracilis se presenta en el primer y segundo día de antesis ya que se produjeron significativamente más semillas que en los días subsecuentes, evidenciando que el periodo de receptividad de las flores es determínate para la producción de semillas y que corresponde con la presencia de líquido estigmático, como se ha sugerido para otras especies del género (Capperino \& Schneider 1985, Wiersema 1988, Orban \& Bouharmont 1995). El líquido estigmático tiene una compleja constitución química, probablemente participa en la receptividad y reconocimiento del polen, así como en la nutrición y llegada del tubo polínico al micrópilo (Orban \& Bouharmont 1995), además se sugiere que su volumen podría estar correlacionado con la cantidad y el tamaño de las semillas (Capperino \& Schneider 1985). En N. gracilis, como en $N$. mexicana y $N$. capensis (Capperino \& Schneider 1985, Orban \& Bouharmont 1995) este líquido se produce por las papilas multicelulares que recubren la superficie del disco y aunque su volumen disminuye a lo largo de la antesis y no es tan abundante como en otras especies (Capperino \& Schneider 1985), es suficiente para que insectos de los géneros Allograpta y Asemosyrphus se ahoguen en él en los primeros días de floración.

Sin embargo, mantener los estigmas receptivos el segundo día de antesis, al mismo tiempo que se liberan granos de polen, también puede aumentar las posibilidades de autofecundación (Wiersema 1988, Endress 2010). La sobreposición de las fases sexuales en el segundo día de antesis en $N$. gracilis, y en algunos casos hasta inicios del tercer día, indica una protoginia incompleta (Çetİnbaș \& Ünal 2014) y señala la oportunidad para la fecundación sin la necesidad de otro individuo. Se ha sugerido que extender la función femenina al segundo día de antesis es una de las estrategias de autogamia que algunas especies de Nymphaea utilizan para producir semillas sin polinización cruzada (Wiersema 1988).

A nivel poblacional, la atracción de un mayor número de polinizadores también podría facilitarse exponiendo un mayor número de flores. Endress (2010) sugiere que en las poblaciones de Nymphaeaceae un individuo produce varias flores en cada estación, pero usualmente solo mantiene abierta una flor en un momento determinado. En $N$. gracilis las plantas producen hasta cinco flores en la estación de crecimiento y algunas mantienen simultáneamente más de una flor en diferentes estados de antesis, como lo muestra el índice de sincronía entre la fase masculina y femenina. Al mantener simultáneamente más de una flor y exponerlas por más tiempo, automáticamente aumenta la exposición de la fase masculina, pero al superponer las fases de la antesis se posibilita la autofecundación por geitonogamia ya que los insectos que visitan una flor funcionalmente masculina de tercer, cuarto o quinto día, también pueden visitar flores de la misma planta pero que se encuentran en el primer y segundo día, donde son funcionalmente femeninas. Una cierta cantidad de geitonogamia es prácticamente inevitable en plantas autocompatibles que producen varias flores en antesis al mismo tiempo (Lloyd \& Schoen 1992), pero dado que no proporciona una garantía reproductiva y puede generar importantes pérdidas de polen y semillas, no se considera un mecanismo adaptativo (Eckert 2000). La polinización artificial mostró la posibilidad de geitonogamia en la especie, sin embargo, su presencia en condiciones naturales debe evaluarse.

Visitantes florales. Las características florales usualmente se asocian con el tipo de polinización, las flores diurnas y brillantes de algunas especies de los subgéneros Brachyceras, Anecphya y Nymphaea se relacionan con síndromes de polinización por insectos generalistas como himenópteros o dípteros (Prance \& Anderson 1976, $\underline{\text { Schneider 1982, Capperino \& Schneider 1985, Wiersema }}$ 
1988). En N. gracilis se observa una baja diversidad de visitantes florales; sus flores amplias, sutilmente fragantes, con abundante polen, disco estigmático y estambres amarillos contrastantes con el perianto blanco, atraen diferentes especies de dípteros e himenópteros, pero sobre todo a Apis mellifera.

Apis mellifera también es un polinizador de Nymphaea ampla (Salisb.) DC., N. mexicana, N. naucalli y N. odorata, aunque en cada caso presenta diferentes tasas de efectividad (Schneider \& Chaney 1981, Capperino \& Schneider 1985, Barrios \& Ramírez 2008, Williams et al. 2010). Por ejemplo, en $N$. ampla, otra especie del subgénero Brachyceras con rasgos florales similares a N. gracilis, esta abeja es un visitador habitual que parece facilitar la autogamia directa más que la fecundación cruzada (Barrios \& Ramírez 2008). Las frecuentes visitas a las flores de $N$. gracilis y la recolecta de grandes cantidades de polen por parte de $A$. mellifera la hacen el visitador más eficiente e importante de todos los insectos que llegan a las flores de esta hidrófita. Sin embargo, sus cortas visitas a las flores de primer día suponen baja eficacia en la polinización, por lo que su comportamiento y efectividad de polinización, en términos de la cantidad de semillas producidas, debe evaluarse detalladamente.

Después de A. mellifera, Allograpta obliqua y Asemosyrphus sp. fueron los insectos que más visitaron las flores de $N$. gracilis; especies de Allograpta también se han observado en flores de $N$. odorata (Schneider \& Chaney 1981) y $N$. elegans (Schneider 1982) aunque son poco frecuentes. Los datos indican que la carga de polen y en general la contribución de estos dos insectos a la polinización de la especie es muy baja, sin embargo, su frecuente muerte en el líquido estigmático pocas horas después de la apertura floral podría estar contribuyendo a la fecundación cruzada. Diferentes autores (Schneider \& Chaney 1981, Schneider 1982) proponen que la acumulación del líquido sobre el estigma funciona como una trampa, que junto con la apertura parcial de las flores durante el inicio de la antesis podría favorecer la polinización cruzada al lavar el polen del cuerpo de los insectos que tienen contacto o que terminan ahogados en él.

Sistema reproductivo. El sistema reproductivo en una planta se puede inferir por la relación polen:óvulo (Cruden 1977, 2000). De acuerdo con esto, la relación polen: óvulos calculada para $N$. gracilis indica xenogamia facultativa. La xenogamia facultativa es una estrategia frecuente en ambientes perturbados o estados sucesionales tempranos donde las plantas presentan adaptaciones que favorecen la interacción con polinizadores y la fecundación cruzada (Cruden 1977). Pero, además, regularmente son plantas autocompatibles y muchas se autopolinizan cuando las flores se cierran, de tal forma que la autopolinización ocurre en ausencia o además de la fecundación cruzada como un mecanismo para asegurar la reproducción (Cruden 1977, 2000).

Otros atributos de $N$. gracilis asociados con el sistema reproductivo mixto se observan en las inversiones de biomasa calculadas. En primer lugar, la relación de biomasa androceo:gineceo en $N$. gracilis $(1.4: 1 \mathrm{~g})$ indica una asignación ligeramente mayor al androceo; este valor puede considerarse intermedio entre la gama de autogamia y xenogamia absoluta y sugiere que la planta puede presentar autofecundaciones, pero que algunos incrementos en la asignación de recursos a la función masculina podrán facilitar la fecundación cruzada (Lovett-Doust \& Cavers 1982). Los valores de biomasa masculina relativa a la biomasa reproductiva de las flores de $N$. gracilis $(\mathrm{M} 1=0.57)$ reafirman este hecho, ya que usualmente valores bajos en este índice se han relacionado a incrementos en los niveles de autocompatibilidad y viceversa (Barrios \& Ramírez 2008).

En segundo lugar, la baja inversión en estructuras sexuales de prefertilización que muestra el índice de inversión de biomasa sexual floral sobre la biomasa vegetativa de $N$. gracilis (1.2:1 g), sugiere que la planta podría estar asegurando alguna polinización ya que no está invirtiendo mucho en estructuras de atracción, como ha sido sugerido por Raimúndez \& Ramírez (1998) para algunas hierbas perennes. Entonces, si los recursos de N. gracilis no se están usando para estructuras de atracción podrían dirigirse a otras funciones como frutos y/o semillas.

La relación peso del fruto: peso de la flor $(8.41 \mathrm{~g})$ indica que los frutos de $N$. gracilis no son costosos comparados con los frutos de herbáceas perennes (Ramírez 1993) y por lo tanto su producción es segura, esto debido a que una pequeña ganancia de peso entre la transición de flor a fruto se asocia con mayor éxito en la producción de frutos, mientras que un incremento grande de peso implica menor fructificación (Ramírez \& Berry 1993, Raimúndez \& Ramírez 1998).

Por su parte el costo de inversión materna: progenie en $N$. gracilis $(0.32 \mathrm{~g})$ indica que la biomasa asignada a la producción de semillas es alta en comparación con la asignada al pericarpo (Ramírez 1993, Ramírez \& Berry 1993). Esto significa que el conflicto materno:progenie es mínimo ya que la progenie tiene los recursos suficientes para desarrollarse y los recursos maternos se administran de tal forma que la mayor cantidad de semillas se empaquetan en frutos de bajo costo (Raimúndez \& Ramírez 1998, Barrios \& Ramírez 2008). En especies con muchas semillas, como es el caso de $N$. gracilis, esto puede ayudar al éxito reproductivo ya que se maximiza la producción semillas (Ramírez \& Berry 1993). Además, parece estar relacionado al transporte de polen en masa, en donde la baja diversidad genética de la progenie disminuye la rivalidad ( 
Berry 1993), una situación que podría estar sucediendo a partir del segundo día de antesis de $N$. gracilis, con la liberación de un gran número de granos de polen de las anteras exteriores que potencialmente podrían fecundar al estigma propio.

Tratamientos de polinización y depresión endogámica. Con los cruzamientos experimentales manuales se determinó que $N$. gracilis presenta un sistema de cruzamiento mixto, autocompatibilidad y autogamia. Es decir, puede polinizarse por polen de diferente origen o por su mismo polen, ya sea de sus propias anteras (autopolinización) o de flores de la misma planta (geitonogamia).

En general los tratamientos de polinización manual resultaron ser más eficientes que los de polinización natural en $N$. gracilis. En la polinización asistida la producción de semillas fue mayor debido a que se aseguró la llegada de polen a los estigmas, sugiriendo que para la especie los polinizadores son importantes. No obstante, la producción de semillas por xenogamia manual fue mayor que la de los tratamientos control y xenogamia natural, indicando una baja transmisión de polen en condiciones naturales, probablemente asociada a una baja disponibilidad y/o eficiencia de los polinizadores, como también se ha reportado para otras especies del género (Barrios \& Ramírez 2008).

Una disminución en los servicios de los polinizadores tiene efectos directos en la reproducción y permanencia de las especies ya que disminuye la cantidad y calidad de frutos y semillas (Agren 1996). Sin embargo, para evitar depender completamente de los polinizadores algunas especies tropicales de amplia distribución de Nymphaeaceae emplean más de una alternativa reproductiva y la principal estrategia es la autogamia (Wiersema 1988). El porcentaje de frutos formados por autogamia confirma esta posibilidad e indica que $N$. gracilis está asegurando su reproducción aun cuando los polinizadores no están siendo efectivos (limitación reproductiva por polinizadores $=0.4$ ), mientras que el índice de autogamia (IAS $=0.88$ ) apunta a que la autopolinización en condiciones naturales tiene altas posibilidades de realizarse a nivel intraflor. Si bien la autopolinización se previene el primer día de la floración, la protoginia incompleta que $N$. gracilis muestra en el segundo día de antesis permite que los granos de polen lleguen al estigma propio cuando este aún es receptivo. Esta conducta se ajusta a la denominada autofecundación tardía (Lloyd \& Schoen 1992, Goodwillie \& Weber 2018), no obstante, la consideración precisa de su presencia y los beneficios que representa para la reproducción exitosa de $N$. gracilis requiere de análisis más rigurosos.

La capacidad de autofecundación presumiblemente evolucionó como una ventaja al garantizar la reproducción, pero esta ventaja se contrarresta por un mayor riesgo de depresión por endogamia (Charlesworth 2006). De acuerdo con los resultados, en la población analizada la progenie de fecundación cruzada supera en número a la progenie producida por autofecundación. Sin embargo, la presencia de endogamia alerta sobre el riesgo que podría representar el aumento de la autogamia en la especie, y requiere explorar el efecto de la autofecundación en el desempeño de la descendencia, por ejemplo, en la tasa de germinación y supervivencia de plántulas.

El sistema mixto de reproducción en $N$. gracilis con altos niveles de autocompatibilidad, evidenciados por la alta producción de semillas por geitonogamia y autogamia, podría estar favoreciendo la permanencia de la población y garantizar la descendencia, pero sin descartar la posibilidad de fecundaciones cruzadas que eviten la depresión por autofecundación. Esto se explicaría considerando que, si bien la polinización cruzada puede representar la mejor opción debido a que disminuye la probabilidad de homocigosis (Wells 1979), la posibilidad de autopolinización puede resultar favorable en términos de asegurar la reproducción (Cruden 1977, 2000) en condiciones en las que los donantes de polen coespecíficos sean escasos o debido a una deficiencia en los servicios de los polinizadores, tal como ha sido planteado en el contexto de la hipótesis de aseguramiento reproductivo (Charlesworth 2006).

Al menos 10 especies de Nymphaea utilizan la autogamia como estrategia reproductiva, cuatro pertenecen al subgénero Brachyceras como N. gracilis (Wiersema 1988, Povilus et al. 2014). La adquisición de esta estrategia en el género y especialmente en el subgénero Brachyceras, se ha relacionado con la distribución tropical de las especies (Wiersema 1988). Si las semillas superan las barreras iniciales de dispersión y las plántulas se establecen, la supervivencia de las plantas en nuevos ambientes estaría principalmente vinculada con la capacidad de la especie para maximizar la producción de semillas en ausencia de polinizadores potenciales y al mismo tiempo producir progenie adaptada a las condiciones ambientales donde creció la planta madre. Del mismo modo, bajo situaciones de colonización, la autogamia eliminaría la necesidad de un segundo individuo para llevar a cabo la polinización, aunque también podría hacerlo por propagación vegetativa o geitonogamia en individuos con varias flores (Wiersema 1988).

La predisposición a la autofecundación es una estrategia que probablemente ha evolucionado varias veces en Nymphaea y puede tener profundos efectos sobre la dinámica de la población y, por lo tanto, en la historia evolutiva de la especie (Povilus et al. 2014). En poblaciones pequeñas, fragmentadas y con pocos individuos, la autocompatibilidad puede ser ventajosa para garantizar la 
producción de frutos y semillas, aunque algunos autores consideran que contribuye a la desaparición de las especies, dado que incrementa las tasas de depresión endogámica (Brook et al. 2002, Povilus et al. 2014). En N. gracilis, este hecho puede agravarse por la distribución limitada que tiene la especie, la sobreexplotación y la baja densidad que imponen los hábitats en los que se desarrolla. Los problemas ambientales que prevalecen en la Cuenca del Río Lerma han degradado profundamente los ecosistemas acuáticos y han fragmentado las poblaciones de ésta y otras hidrófitas (Lot \& Zepeda 2009), lo que puede estar causando una erosión genética provocada por la baja disponibilidad de compañeros potenciales y genéticamente diferentes, o por baja disponibilidad de polinizadores que aseguren la fertilización cruzada (Charlesworth 2006). Aun cuando los niveles de depresión por endogamia son bajos, si los servicios de los polinizadores disminuyen a tal grado que no puedan mantener suficientes tasas de fecundación cruzada, la reproducción y permanencia de $N$. gracilis dependerán de la autogamia, geitonogamia y/o reproducción vegetativa, lo que hará que su diversidad genética baje y como consecuencia la capacidad para adaptarse a los cambios ambientales. Alternativas para disminuir este efecto podrían ser la polinización asistida, el incremento de poblaciones e individuos con carga genética diferente a través de intervenciones artificiales, así como la restauración de cuerpos de agua y el incremento de su conectividad.

Este estudio muestra que $N$. gracilis tiene un despliegue floral que varía a lo largo de la antesis, sus atributos estructurales y funcionales sustentan un sistema de apareamiento mixto y protógina incompleta, como se ha observado en otros integrantes del subgénero Brachyceras. El deterioro y disminución progresiva de su hábitat y de interacciones con polinizadores potenciales, puede conducir a procesos endogámicos generalizados que coloquen a $N$. gracilis en un riesgo de extinción mayor. El entendimiento de la biología floral desde una perspectiva integral y considerando el efecto de las condiciones ambientales locales, los atributos demográficos, los rasgos de historia de vida y el tipo y eficiencia de las comunidades de polinizadores son esenciales y urgentes para plantear estrategias viables de conservación de esta especie mexicana.

\section{Agradecimientos}

A Guillermo Morales-Merino y Javier Manjarrez por el apoyo en el trabajo de campo, a la Dra. Karina Cuevas Yañez por la determinación de los visitantes florales, a la Universidad Autónoma del Estado de México por el financiamiento y las facilidades otorgadas. A los revisores anónimos y el editor asociado por las aportaciones que enriquecieron el documento final.

\section{Literatura citada}

Ågren J, Schemske DW. 1993. Outcrossing rate and inbreeding depression in two annual monoecious herbs, Begonia hirsuta and B. semiovata. Evolution 47: 125-135. DOI: https://doi.org/10.1111/j.1558-5646.1993. tb01204.x

Agren J. 1996. Population size, pollinator limitation, and seed set in the self-incompatible herb Lythrum salicaria. Ecology 77: 1779-1790. DOI: https://doi.org/10.2307/ 2265783

Augspurger CK. 1983. Phenology, flowering synchrony and fruit set of six neotropical shrubs. Biotropica 15: 257-267. DOI: https://doi.org/10.2307/2387650

Barrios Y, Ramírez N. 2008. Depresión por exogamia y biología reproductiva de Nymphaea ampla (Salisb.) DC. (Nymphaeaceae). Acta Botanica Venezuelica 31: 539-556.

Begum AH, Ghosal KK, Chattopadhyay TK. 2010. Comparative morphology and floral biology of three species of the genus Nymphaea from Bangladesh. Bangladesh Journal of Botany 39: 179-183. DOI: https:// doi.org/10.3329/bjb.v39i2.7478

Borsch T, Löhne C, Mbaye SM, Wiersema JH. 2011. Towards a complete species tree of Nymphaea: shedding further light on subg. Brachyceras and its relationships to the Australian water-lilies. Telopea 13: 193-217. DOI: https://doi.org/10.7751/telopea20116014

Brook BW, Tonkyn DW, O’Grady JJ, Frankham R. 2002. Contribution of inbreeding to extinction risk in threatened species. Conservation Ecology 6: 16. DOI: https://doi.org/10.5751/ES-00387-060116

Calderón de Rzedowski G, Rzedowski J. 2010 Flora fanerogámica del Valle de México. México: Instituto de Ecología A.C. y Comisión Nacional para el Conocimiento y Uso de la Biodiversidad. ISBN: 978-607-7607-36-6.

Capperino ME, Schneider EL. 1985. Floral biology of Nymphaea mexicana Zucc. (Nymphaeaceae). Aquatic Botany 23: 83-93. DOI: https://doi.org/10.1016/0304 -3770(85)90022-1

Castro S, Silveira P, Navarro L. 2008. How flower biology and breeding system affect the reproductive success of the narrow endemic Polygala vayredae Costa (Polygalaceae). Botanical Journal of the Linnean Society 157: 67-81. DOI: https://doi.org/10.1111/j.1095-8339. 2008.00784.X

Çetİnbaş A, Ünal M. 2014. An overview of dichogamy in angiosperms. Research in Plant Biology 4: 09-27.

Charlesworth D. 2006. Evolution of plant breeding systems. Current Biology 16: 726-735. DOI: https://doi.org/ $\underline{10.1016 / \text { j.cub. } 2006.07 .068}$ 
Conard H. 1905. The water lilies: a monograph of the genus Nymphaea. Washington, D.C.: Carnegie Institution of Washington. ISBN-13: 978-0948697173. DOI: https:// doi.org/10.5962/bhl.title.51290

Cruden RW. 1977. Pollen-ovule ratios: a conservative indicator of breeding systems in flowering plants. Evolution 31: 32-46. DOI: https://doi.org/10.2307/24 07542

Cruden RW. 2000. Pollen grains: why so many? Plant Systematics and Evolution 222: 143-165. https:// www.jstor.org/stable/23644332 (acceso mayo 12, 2020).

Dafni A. 1992. Pollination ecology: A practical approach. New York, USA: Oxford University Press. ISBN: 9780199632992.

Dafni A, Pacini E, Nepi M. 2005. Pollen and stigma biology. In: Dafni A, Kevan PG, Husband B, eds. Practical Pollination Biology. Canada: EnviroQuest Ltd. ISBN:13: 9780968012307.

Eckert CG. 2000. Contributions of autogamy and geitonogamy to self-fertilization in mass flowering clonal plant. Ecology 81: 532-542. DOI: https://doi.org/ $\underline{10.2307 / 177446}$

Endress PK. 2001. The flowers in extant basal angiosperms and inferences on ancestral flowers. International Journal of Plant Sciences 162: 1111-1140. DOI: https:// doi.org/10.1086/321919

Endress PK. 2010. The evolution of floral biology in basal angiosperms. Philosophical Transactions of the Royal Society B Biological Sciences 365: 411-421. DOI: https:// doi.org/10.1098/rstb.2009.0228

Escaravage N, Wagner J. 2004. Pollination effectiveness and pollen dispersal in a Rhododendron ferrugineum (Ericaceae) population. Plant Biology 6: 606-615. DOI: https://doi.org/10.1055/s-2004-821143

GEM [Gobierno del Estado de México]. 2011. Atlas de la Cuenca del Río Lerma en el Estado de México Toluca, México: Fondo Editorial Estado de México. ISBN: 978-607-495-153-0.

Goodwillie C, Weber JJ. 2018. The best of both worlds? A review of delayed selfing in flowering plants. American Journal of Botany 105: 641-655. DOI: https://doi.org/ $\underline{10.1002 / a j b 2.1045}$

Harder LD, Barrett SCH. 2006. Ecology and Evolution of Flowers. New York: Oxford University Press. ISBN: 978-0-19-857085-1.

Hirthe G, Porembski S. 2003. Pollination of Nymphaea lotus (Nymphaeaceae) by rhinoceros beetles and bees in Northeastern Ivory Coast. Plant Biology 5: 670-676. DOI: https://doi.org/10.1055/s-2003-44717

IBM. 2015. IBM SPSS Statistics for Windows, Version 23.0. Armonk, NY: IBM Corp.
Kearns CA, Inouye DW. 1993. Techniques for pollination biologists. Niwot, Colorado: University Press of Colorado. ISBN: 978-0-87081-281-1.

Lloyd DG, Schoen DJ. 1992. Self- and cross-fertilization in plants. I. Functional dimensions. International Journal of Plant Sciences 153: 358-369.

Lot A, Novelo A, Olvera M, Ramírez-García P. 1999. Catálogo de angiospermas acuáticas de México. México, DF: Instituto de Biología. Universidad Nacional Autónoma de México. ISBN: 9683679284.

Lot A, Zepeda C. 2009. Plantas acuáticas. In: Ceballos G, List R, Garduño G, López- Cano R, MuñozcanoQuintanar MJ, Collado E, San Román JE. eds. La diversidad biológica del Estado de México. Toluca, México: Biblioteca Mexiquense del Bicentenario, Gobierno del Estado de México, pp. 229-241. ISBN: 9789708260633.

Lovett-Doust J, Cavers PB. 1982. Biomass allocation in hermaphrodite flowers. Canadian Journal of Botany 60: 2530-2534. DOI: https://doi.org/10.1139/b82-306

Maia ACD, de Lima CT, Navarro DMDAF, Chartier M, Giulietti AM, Machado IC. 2014. The floral scents of Nymphaea subg. Hydrocallis (Nymphaeaceae), the New World night-blooming water lilies, and their relation with putative pollinators. Phytochemistry 103: 67-75. DOI: https://doi.org/10.1016/j.phytochem.2014.04.007

Navarro L, Guitián J. 2002. The role of floral biology and breeding system on the reproductive success of the narrow endemic Petrocoptis viscosa Rothm. (Caryophyllaceae). Biological Conservation 103: 125-132. DOI: https://doi.org/10.1016/S0006-3207(01) $\underline{00108-2}$

Ne'eman G, Dafni A, Potts SG. 1999. A new pollination probability index (PPI) for pollen load analysis as a measure for pollination effectiveness of bees. Journal of Apicultural Research 38: 19-23. DOI: https://doi.org/ $\underline{10.1080 / 00218839.1999 .11100991}$

Novelo A, Bonilla-Barbosa J. 1999. Nymphaeaceae. Flora del Bajío y de regiones adyacentes 92: 1-120.

Orban I, Bouharmont J. 1995. Reproductive biology of Nymphaea capensis Thunb. var. zanzibariensis (Casp.) Verdc. (Nymphaeaceae). Botanical Journal of the Linnean Society 119: 35-43. DOI: https://doi.org/10. 1111/j.1095-8339.1995.tb00727.x

Pinilla-Gallego MS, Nates-Parra G. 2015. Visitantes florales y polinizadores en poblaciones silvestres de agraz (Vaccinium meridionale) del bosque andino colombiano. Revista Colombiana de Entomología 41: 112-119.

Povilus RA, Losada JM, Friedman WE. 2014. Floral biology and ovule and seed ontogeny of Nymphaea thermarum, a water lily at the brink of extinction with potential as a model system for basal angiosperms. Annals of Botany 115: 211-226. DOI: https://doi.org/ $\underline{10.1093 / \mathrm{aob} / \mathrm{mcu} 235}$ 
Prance GT, Anderson AB. 1976. Studies of the floral biology of neotropical Nymphaeaceae. Acta Amazonica 6: 163-170. DOI: https://doi.org/10.1590/1809-439219 $\underline{76062163}$

Primack RB. 1985. Longevity of individual flowers. Annual Review of Ecology and Systematics 16: 15-37. DOI: https://doi.org/10.1146/annurev.es.16.110185.000311

Raimúndez UE, Ramírez N. 1998. Estrategia reproductiva de una hierba perenne: Hypoxis decumbens (Hypoxidaceae). Revista de Biología Tropical 46: 555-565.

Ramírez N. 1993. Producción y costo de frutos y semillas entre formas de vida. Biotropica 25: 46. DOI: https:// doi.org/10.2307/2388978

Ramírez N, Berry P. 1993. Producción y costo de frutos y semillas relacionados a los tipos morfológicos de frutos, unidad de dispersión y síndromes de dispersión. Ecotrópicos 6: 42-61.

Ramsey M. 1993. Floral morphology, biology and sex allocation in disjunct populations of Christmas bells (Blandfordia grandiflora, Liliaceae) with different breeding systems. Australian Journal of Botany 41: 749-762. DOI: https://doi.org/10.1071/BT9930749

Rivera-Hutinel A, Acevedo-Orellana F. 2017. Biología floral y reproductiva de Escallonia pulverulenta (Ruiz et Pav.) Pers. (Escalloniaceae) y su relación con los visitantes florales. Gayana Botanica 74: 82-93. DOI: https://doi.org/10.4067/S0717-66432017005000322

Rodríguez-Pérez J. 2005. Breeding system, flower visitors and seedling survival of two endangered species of Helianthemum (Cistaceae). Annals of Botany 95: 1229-1236. DOI: https://doi.org/10.1093/aob/mci137

Ruiz-Zapata T, Kalin-Arroyo M. 1978. Plant reproductive ecology of secondary deciduous forest in Venezuela. Biotropica 10: 221-230. DOI: https://doi.org/10.2307/ 2387907

Sandoval-Ortega MH. 2020. Contribución al conocimiento de Nymphaea gracilis Zucc. en Aguascalientes, México. Tecnociencia Chihuahua 14: 1-6.

SAS. 2013. SAS/Stat user's guide v. 9.4. SAS Institute Inc, Cary, NC, USA.

Schemske DW, Husband BC, Ruckelshaus MH, Goodwillie C, Parker IM, Bishop JG. 1994. Evaluating approaches to the conservation of rare and endangered plants. Ecology 75: 584-606. DOI: https://doi.org/10.2307/ $\underline{1941718}$

\footnotetext{
Editor de sección: Alejandro Zavala Hurtado
}

Contribución de autores: $\mathrm{CZG}$, diseño, análisis y escritura del manuscrito. ECM, trabajo de campo, análisis y escritura del manuscrito. CBA, análisis, redacción de discusión y revisión del manuscrito. MFC, asesoría, redacción de discusión y revisión del manuscrito.
Schneider EL. 1982. Notes on the floral biology of Nymphaea elegans (Nymphaeaceae) in Texas. Aquatic Botany 12: 197-200. DOI: https://doi.org/10.1016/ 0304-3770(82)90014-6

Schneider EL, Chaney T. 1981. The floral biology of Nymphaea odorata (Nymphaeaceae). Southwestern Naturalist 26: 159-165. DOI: https://doi.org/10.2307/ $\underline{3671112}$

SEMARNAT [Secretaría del Medio Ambiente y Recursos Naturales]. 2010. Norma Oficial Mexicana NOM-059SEMARNAT-2010, Protección ambiental - Especies nativas de México de flora y fauna silvestres - Categorías de riesgo y especificaciones para su inclusión, exclusión o cambio - Lista de especies en riesgo. Diario Oficial de la Federación. 2da Sección, 30 de diciembre de 2010.

Sokal RR, Rohlf FJ. 2012. Biometry the principles and practice of statistics in biological research $4 \mathrm{a}$ ed. New York, USA: W.H. Freeman and Company. ISBN-13: 978-0-7167-8604-4.

van der Velde G. 1986. Developmental stages in the floral biology S.L. of dutch Nymphaeaceae (Nymphaea alba L., Nymphaea candida Presl, Nuphar lutea (L.) Sm.). Acta Botanica Neerlandica 35: 111-113. DOI: https:// doi.org/10.1111/j.1438-8677.1986.tb00467.x

Vogel S. 1990. The role of scent glands in pollination: on the structure and function of osmophores. Washington, D.C.: Smithsonian Institution Libraries and National Science Foundation. ISBN-10: 9999962882.

Wells H. 1979. Self-fertilization: Advantageous or deleterious? Evolution 33: 252-255. DOI: https://doi.org/ $\underline{10.2307 / 2407381}$

Wiersema JH. 1988. Reproductive biology of Nymphaea (Nymphaeaceae). Annals of the Missouri Botanical Garden 75: 795-804. DOI: https://doi.org/10.2307 / 2399367

Williams JH, McNeilage RT, Lettre MT, Taylor ML. 2010. Pollen tube growth and the pollen-tube pathway of Nymphaea odorata (Nymphaeaceae). Botanical Journal of the Linnean Society 162: 581-593. DOI: https:// doi.org/10.1111/j.1095-8339.2010.01039.x

Zepeda-Gómez C. 2017. Nymphaeaceae. In: Lot A. ed. Plantas acuáticas mexicanas, una contribución a la flora de México. Vol. II Dicotiledóneas Parte 1. México, D.F.: Instituto de Biología. Universidad Nacional Autónoma de México. pp. 139-154. ISBN: 9786073000574. 
Apéndice 1. Fórmulas empleadas para el cálculo de los índices de sincronía, polinización, biomasa masculina, inversión materna y estrategias reproductivas.

\begin{tabular}{|c|c|c|c|}
\hline Nombre & Formula & Significado & Autor \\
\hline Índice de sincronía & $x_{i}=\left[\frac{1}{(n-1)}\right]\left(\frac{1}{f_{i}}\right) \Sigma e_{j} \neq i$ & $\begin{array}{l}n=\text { número de flores de la muestra, } e_{j \neq i}=\text { número de } \\
\text { días cuando las flores } i \text { y j son receptivas y/o producen } \\
\text { polen sincrónicamente, } f i=\text { número de días que la flor } \\
i \text { estuvo abierta. }\end{array}$ & $\frac{\text { Augspurger }}{(1983)}$ \\
\hline $\begin{array}{l}\text { Índice de } \\
\text { Probabilidad de } \\
\text { Polinización }\end{array}$ & $\mathrm{PPI}=\mathrm{PCP} \times \mathrm{PBP}$ & $\begin{array}{l}\mathrm{PCP}=\text { proporción media de polen de } N \text {. gracilis en } \\
\text { cada especie de insecto, } \mathrm{P} B \mathrm{P}=\text { proporción de } \\
\text { individuos de una especie de insecto que trasportan } \\
\text { polen de } N \text {. gracilis. }\end{array}$ & $\underline{\text { Ne'eman et al. }}$ \\
\hline $\begin{array}{l}\text { Valor de Importancia } \\
\text { de Polinización }\end{array}$ & $\mathrm{PIV}=\mathrm{PE} \times \mathrm{VR} \times \mathrm{C} \times \mathrm{PCC}$ & $\begin{array}{l}\text { PE (eficiencia básica de polinización) evalúa el } \\
\text { comportamiento de los visitantes florales sumando } \\
\text { cuatro parámetros: 1) núm. de flores visitadas/min } \\
\text { (calificado entre } 0 \text { y } 0.3 \text { ); 2) presencia o ausencia en } \\
\text { todos los puntos de muestreo (calificado entre } 0 \text { y } 0.3 \text { ), } \\
\text { 3) contacto de las patas con el estigma (calificado } \\
\text { entre } 0 \text { y } 0.2 \text { ), 4) contacto del abdomen y tórax con el } \\
\text { estigma (calificado entre } 0 \text { y } 0.2 \text { ). VR (tasa de visitas) } \\
=\text { porcentaje total de las visitas de cada especie con } \\
\text { respecto al total de visitas registradas de todos los } \\
\text { insectos. C (constancia) = proporción promedio de } \\
\text { polen de } N \text {. gracilis en la muestra de polen. PCC } \\
\text { (capacidad de trasporte de polen) }=\text { proporción de } \\
\text { polen de } N \text {. gracilis trasportado por cada especie de } \\
\text { visitante del total de polen de } \\
N \text {. gracilis llevado por todos los visitantes. }\end{array}$ & $\begin{array}{l}\frac{\text { Escaravage \& }}{\text { Wagner }(2004) \text { y }} \\
\underline{\text { Pinilla-Gallego \& }} \\
\underline{\text { Nates-Parra }} \\
\underline{\underline{(2015)}}\end{array}$ \\
\hline $\begin{array}{l}\text { Índice de Importancia } \\
\text { de Polinización }\end{array}$ & $P \mathrm{II}=(\mathrm{PIV} / \Sigma \mathrm{PIV}) \times 100$ & PIV = Valor de importancia de polinización. & $\begin{array}{l}\text { Escaravage \& } \\
\text { Wagner }(2004)\end{array}$ \\
\hline $\begin{array}{l}\text { Biomasa masculina } \\
\text { relativa }\end{array}$ & $\mathrm{M} 1=a /((a+g))$ & $\begin{array}{l}a=\text { peso seco promedio del androceo, } g=\text { peso seco } \\
\text { promedio del gineceo. }\end{array}$ & $\underline{\text { Ramsey (1993) }}$ \\
\hline $\begin{array}{l}\text { Biomasa masculina } \\
\text { post-cigótica }\end{array}$ & $\mathrm{M} 2=a /((a+s))$ & $\begin{array}{l}a=\text { peso seco promedio del androceo, } s=\text { peso seco } \\
\text { promedio de las semillas. }\end{array}$ & $\underline{\text { Ramsey (1993) }}$ \\
\hline $\begin{array}{l}\text { Índice de inversión } \\
\text { materna: progenie }\end{array}$ & Inv. mat. : prog. $=(f-s) / s$ & $\begin{array}{l}f=\text { peso promedio del fruto, } s=\text { peso promedio de } \\
\text { semillas por fruto. }\end{array}$ & $\begin{array}{l}\text { Barrios \& } \\
\underline{\text { Ramírez (2008). }}\end{array}$ \\
\hline $\begin{array}{l}\text { Tasa de fecundidad } \\
\text { cruzada }\end{array}$ & $\begin{array}{c}\mathrm{IFC}=1-s \\
s=W s /((W x+W s))\end{array}$ & $\begin{array}{l}s=\text { tasa de autofecundación, } W s=\text { producción de } \\
\text { frutos o semillas por autofecundación artificial } \\
\text { (geitonogamia), } W x=\text { producción de frutos o semillas } \\
\text { por fecundación cruzada artificial (xenogamia } \\
\text { manual). }\end{array}$ & $\begin{array}{l}\text { (Lloyd \& Schoen, } \\
\underline{1992} \text { ) }\end{array}$ \\
\hline $\begin{array}{l}\text { Índice de } \\
\text { Incompatibilidad }\end{array}$ & $\mathrm{ISI}=W s / W x$ & $\begin{array}{l}W s=\text { producción semillas por autofecundación } \\
\text { artificial (geitonogamia), } W x=\text { producción semillas } \\
\text { por fecundación cruzada artificial (xenogamia } \\
\text { manual). }\end{array}$ & $\frac{\underline{\text { Ruiz-Zapata \& }}}{\underline{\text { Kalin.Arroyo }}}$ \\
\hline Índice de Autogamia & $\mathrm{IAS}=(P F a) / P F S$ & $\begin{array}{l}P F a=\text { porcentaje de frutos formados por autogamia, } \\
P F s=\text { porcentaje de frutos formados por } \\
\text { autofecundación artificial. }\end{array}$ & $\frac{\frac{\text { Ruiz-Zapata \& }}{\text { Kalin-Arroyo }}}{(1978)}$ \\
\hline $\begin{array}{l}\text { Éxito reproductivo } \\
\text { relativo }\end{array}$ & $\mathrm{ERR}=[((f r) / f l) \times(s /(o))] \times 100$ & $\begin{array}{l}f r=\text { número de frutos, } f l=\text { número de flores, } s=\text { número } \\
\text { de semillas, } o=\text { número de óvulos }\end{array}$ & Dafni (1992) \\
\hline $\begin{array}{l}\text { Rendimiento relativo } \\
\text { de frutos y semillas }\end{array}$ & $\begin{array}{l}\mathrm{RP}=1-W a / W x \text { si } W a \leq W x \\
\mathrm{RP}=W x / W a-1 \text { si } W a>W x\end{array}$ & $\begin{array}{l}W a=\text { producción semillas por autogamia, } W x= \\
\text { producción semillas por fecundación cruzada artificial } \\
\text { (xenogamia manual). }\end{array}$ & $\begin{array}{l}\underline{\text { Agren \& }} \\
\underline{\text { Schemske (1993) }}\end{array}$ \\
\hline
\end{tabular}

